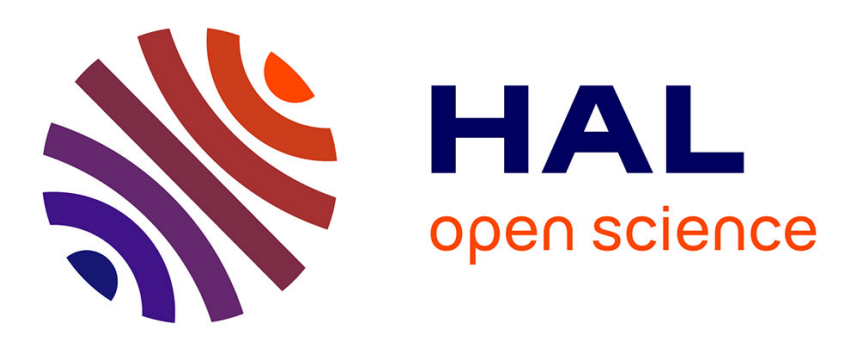

\title{
Randomly-fluctuating heterogeneous continuum model of a ballasted railway track
}

Lucio de Abreu Corrêa, Juan Carlos Quezada, Régis Cottereau, Sofia S Costa D 'Aguiar, Charles Voivret

\section{- To cite this version:}

Lucio de Abreu Corrêa, Juan Carlos Quezada, Régis Cottereau, Sofia S Costa D 'Aguiar, Charles Voivret. Randomly-fluctuating heterogeneous continuum model of a ballasted railway track. Computational Mechanics, 2017, 60 (5), pp.845 - 861. 10.1007/s00466-017-1446-8 . hal-01635950

\section{HAL Id: hal-01635950 https://hal.science/hal-01635950}

Submitted on 16 Nov 2017

HAL is a multi-disciplinary open access archive for the deposit and dissemination of scientific research documents, whether they are published or not. The documents may come from teaching and research institutions in France or abroad, or from public or private research centers.
L'archive ouverte pluridisciplinaire HAL, est destinée au dépôt et à la diffusion de documents scientifiques de niveau recherche, publiés ou non, émanant des établissements d'enseignement et de recherche français ou étrangers, des laboratoires publics ou privés. 


\title{
Randomly-fluctuating heterogeneous continuum model of a ballasted railway track
}

\author{
L. de Abreu Corrêa · J. C. Quezada · R. Cottereau · S. Costa d'Aguiar · C. Voivret
}

Received: date / Accepted: date

\begin{abstract}
This paper proposes a description of a granular medium as a stochastic heterogeneous continuum medium. The heterogeneity of the material properties field recreates the heterogeneous stress field in a granular medium. The stochastic approach means that only statistical information, easily available, is required to construct the model. The heterogeneous continuum model is calibrated with respect to discrete simulations of a set of railway ballast samples. As they are continuum-based, the equilibrium equations can be solved on a large scale using a parallel implementation of an explicit time discretization scheme for the Finite Element Method. Simulations representative of the influence on the environment of the passage of a train on a ballasted railway track clearly show the influence of the heterogeneity. These simulations seem to correlate well with previously unexplained overly damped measurements in the free field.
\end{abstract}

Keywords Ballast railway track - Stochastic heterogeneous material $\cdot$ Spectral Element Method · Granular medium

The spectral element software used for the simulation in this paper is developed jointly by CentraleSupélec, CEA Commissariat à l'Énergie Atomique and Institut de Physique du Globe de Paris. Within the SINAPS@ project, this development benefited from French state funding managed by the National Research Agency under program RNSR Future Investments bearing reference No. ANR-11-RSNR0022-04. The simulations were performed at the Mésocentre of CentraleSupélec-ENS Paris-Saclay.

L. de Abreu Corrêa $\cdot$ R. Cottereau

MSSMat, CNRS, CentraleSupélec, Université Paris-Saclay, France

E-mail: regis.cottereau@centralesupelec.fr

J. C. Quezada

iCUBE, UMR 7347, CNRS, INSA de Strasbourg, France

S. Costa d'Aguiar $\cdot$ C. Voivret

SNCF, Innovation \& Research Dept., France

\section{Introduction}

The ballast is the uppermost layer of the railway track superstructure. It is made of coarse crushed stone, whose size distribution lies in the range $25-50 \mathrm{~mm}$. This ballast layer plays an important role in the transmission and repartition of static and dynamic train loads, the absorption of mechanical and acoustical vibrations, and the drainage of rain water [44]. The noise and vibration impact on the environment can be important for all types of trains: heavy freight coaches, whose low frequency impact in the soil carries far away; high-speed trains, that generate high amplitude excitations; and even tramways (although more rarely on ballasted layers), which stand very close to surrounding buildings. With time and the repeated passage of trains, the ballast layer settles down. This induces a leveling defect of the track and may force the train companies to locally reduce the velocity of the passing trains for the comfort and security of passengers. These defects are therefore regularly controlled, and costly maintenance operations are organized. The modeling of the ballast aims at improving the understanding of the mechanical behavior of the track system and providing clues to mitigate the issues listed above [41,22]. It represents a challenging task because granular materials exhibit various distinct behaviors (such as transition between fluid-like and solid-like behavior) depending on the applied stresses and strains.

The relative displacements between two grains in the ballast layer are in general much larger than the strains within one grain. Models can then be constructed [55] where each grain of the ballast is a rigid body with a complex shape [59, 3]. At this local scale, the static variables are the contact forces and moments between grains and the kinematic variables are the rigid body movements of the grains, and they are related through equilibrium equations and contact laws, such as Coulomb friction law. These models have given rise 
to various numerical implementations, among which the Discrete Element Method (DEM) [24] and the Non-Smooth Contact Dynamics method (NSCD) [65]. In the DEM, the noninterpenetration condition at the interface between two grains is relaxed through a stiff non-linear repulsion law. This gives rise to an explicit scheme in time, which requires very small time steps and needs to handle the changes in the topology, which limits the scalability of parallel implementations on memory-distributed clusters. The NSCD method can deal with multiple contacts and velocity changes within a single time step and reformulates the non-interpenetration condition as a quadratic optimization problem. It yields an implicit scheme, which remains stable for larger time steps, although the scalability of the parallel implementations of these models is not sufficient $[86,101,5]$. The results obtained with these granular approaches are able to reproduce the solid to liquid transition and the inelastic deformations of the ballast $[81,84,11]$ as well as the seemingly random patterns of contact forces $[56,12]$ that can be observed experimentally at that scale [28]. Because of the numerical issues mentioned above, however, these models cannot reproduce dynamical phenomena involving the passage of a train over a large portion of a track. Also, the requirement of inputing a precise geometry [94] and initial position of the seemingly random assembly of grains is straining for most industrial applications, even though large databases of digitized ballast grains have been created [11].

An alternative approach consists in modeling the ballast as a continuous medium, using the stress tensor as the static variable and the displacement field as the kinematical variable. The stress and strain tensors can be derived, respectively, from the contact forces network [111,91,14,67,92, 69] and the displacements of the grains [106,51,18,13,89, $29]$ in discrete simulations, but the relation between the parameters of the continuum and granular models is not obvious. Such continuum models have been developed along various directions $[77,7]$ :

1. phenomenological approaches, that consist in postulating the form of the energy functional, introducing a heuristic constitutive relation, generally non-linear, through observation of experimental results.

2. homogenization approaches, in which an equivalent homogeneous energy functional is derived from mathematical considerations and asymptotic analysis; these approaches can be extended to higher-order continuum approaches (such as Cosserat models [99]), with the introduction of additional degrees of freedom and non-local continuous operators;

Phenomenological approaches have yielded a wide variety of non-linear models, either oriented to the description of granular flows $[43,33,48]$, to that of static pilings [60], including the consideration of wave propagation $[34,54]$. The homogenization of granular structures has been widely studied, for general lattice structures $[64,102,61,74,40]$, random packings of spheres $[17,27,110,20,19,47,2]$, as well as railway ballasted tracks $[62,63,93,12]$.

Both the discrete and continuum approaches are interesting in their own right. The discrete approaches provide very detailed information about the micro-mechanical heterogeneity and behavior of the granular system, but the numerical simulation of large packings is out of reach. The continuum-based methods are more manageable on a large scale but they lose the heterogeneity of realistic force networks, which has been shown to be fundamental to explain many granular phenomena, such as the solid-fluid transition $[70,85,98]$. Because they allow to simulate larger ballasted tracks as well as their surrounding environnement, continuum-based approaches have nevertheless been preferred for dynamic analyses, such as to evaluate the influence of the passing train on the environment. Unfortunately, large amplitude differences are generally found between the simulated and measured accelerations, in particular away from the track $[76,57]$.

The objective of this paper is to explore an intermediate approach. The granular medium is replaced, as in the classical homogenization approaches, by a classical continuum medium, but with a heterogeneous Young's modulus, fluctuating over a characteristic scale equal to the diameter of the ballast grains. Because of the heterogeneity of the material parameter, the stress and strain fields fluctuate within the continuum sample, as in a realistic granular medium. As the model is continuum-based, very efficient parallelized numerical techniques allow to solve large-scale dynamical problems. Although, this would not be sufficient for many types of problems, we concentrate here on a simple linear elastic model, where the Young's modulus is heterogeneous. To simplify the parametrization of that fluctuating modulus, we consider a stochastic model. Hence, the model is parameterized only by statistical information on the sample (average diameter of the particles, mean, variance and autocovariance of the Young's modulus). These parameters have to be experimentally identified, and the inverse problem is described in this paper. We propose an identification technique using the numerical results based on granular samples simulated with a discrete approach (NSCD), rather than actual experimental measurements.

The paper is organized as follows. Next section (Section 2) describes discrete numerical samples of a granular medium, whose normal force distributions can be simulated with the NSCD. These simulations are used to identify the random model of Young's modulus. The following section (Section 3) constitutes the core of the paper, where the randomlyheterogeneous continuum model of the ballast is introduced. Section 4 describes the identification of the parameters of that model, based on the numerical samples described in 
Section 2. Finally, in Section 5, we present large scale simulations of the influence of the passage of a train over a ballasted railway track. The simulation clearly shows the influence of the heterogeneity, in particular through the observation of apparent damping in the free-field response.

Before starting with the bulk of the paper, it should be stated that the model introduced here clearly lacks important features for a granular medium. These features include the dependance of the wave velocity on the applied pressure [73, 60]. However, we focus here on discussing the statement that heterogeneity is essential, even for continuum-based models. More complex constitutive relation and continuum-based models can be derived in forthcoming works.

\section{Discrete model of a granular medium and numerical samples}

This section introduces discrete samples of a granular medium, and describes their force distributions, as estimated with the NSCD. These samples will be used to identify the parameters of the randomly heterogeneous continuum model in Section 4 . The link with the continuum models is performed through equivalent stresses.

\subsection{Description of the discrete numerical samples}

We consider 29 samples of 2700 granular particles, on average, contained in cylindrical boxes with a radius of $R=35 \mathrm{~cm}$ and a height of $H=39 \mathrm{~cm}$ (see Fig. 1). The particles are convex polyhedra with diameters between $2.5 \mathrm{~cm}$ and $5 \mathrm{~cm}$, whose shapes have been obtained by digitalization from real ballast [11]. The 2700 granular particles are randomly drawn from a larger database so the particles are not the same in all samples. An isotropic confinement pressure of $60 \mathrm{kPa}$ is applied on all samples. A vertical load of $63 \mathrm{kN}$ is also applied on the upper face. Gravity is considered within all samples, with particles having a density of $2700 \mathrm{~kg} / \mathrm{m}^{3}$. Contact forces in each of the samples are estimated using the LMGC90 software [1], which is built on the NSCD and considers Coulomb dry friction and mutual exclusion as contact laws. The preparation (creation of the granular assemblies by free fall of randomly-rotated particles in the cylindrical box from grid positions in the air) and simulation of one granular sample takes on average $6200 \mathrm{~s}$ of CPU time.

For each sample, only the contacts contained within a $48 \times 48 \times 32 \mathrm{~cm}^{3}$ hexahedral box are considered, to limit the influence of the boundary conditions. Finally, results from the 29 samples can be aggregated, yielding approximations of the distribution of the contact forces. For instance, Fig. 2 presents the probability distribution function for the normal contact forces normalized by their average value estimated from the 29 samples, and plotted in log-log scale.

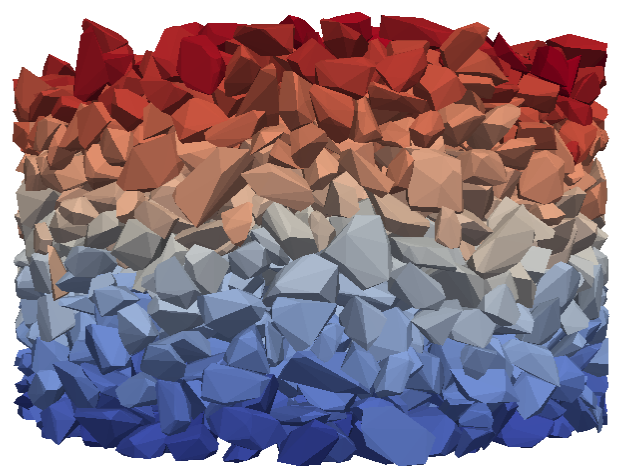

Fig. 1 Example of one of the 29 samples of granular medium simulated with the NSCD.

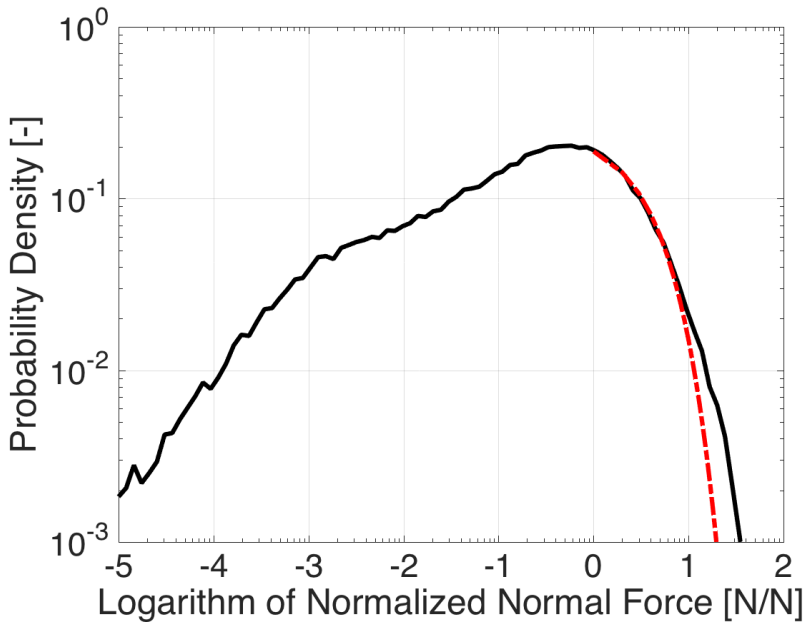

Fig. 2 The probability density function of the logarithm of normal contact force normalized by its average (solid line), and exponential approximation (dashed line, see Eq. (1)).

In the literature, there seems to be a consensus about the exponential distribution for the modeling of the probability distribution of higher-than-average normal forces (socalled strong forces). This consensus is built at the same time over theoretical considerations [23,12,31], experimental evidence $[62,68,105,42]$ and numerical simulations [71, 82]. For the samples considered here, the higher-than-average force distribution is proportional to:

$\mathbb{P}\left(f_{n}\right) \propto \exp \left(\beta \frac{f_{n}}{\left\langle f_{n}\right\rangle}\right)$.

where $\beta=1.13$. Note that the latter value is compatible with the values found in the literature $0.88<\beta<1.71[9,83$, 90], and matches the coefficient of [11], obtained for similar samples. For lower-than-average forces, the situation is not so clear and several models are competing [62,30,42]. In that respect, the size of the particles [62], the shape of the particles [11], and the mean packing fraction [42] seem to be influential.

Finally, the anisotropy of the behavior is reflected in Fig. 3 and well approximated by the following spherical harmonic 


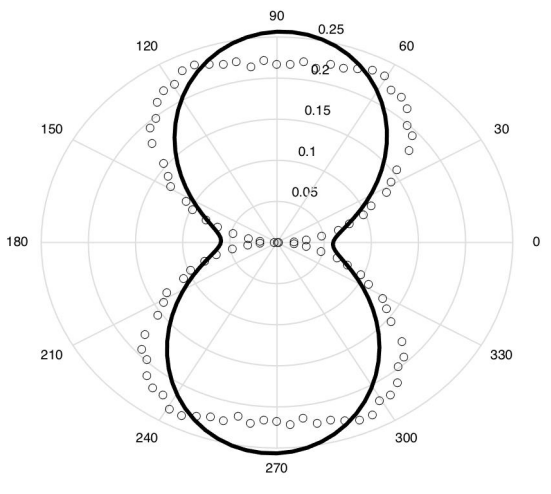

Fig. 3 Anisotropy of the normal contact force distribution, averaged over the 29 discrete samples (in circles), and first spherical harmonic approximation (solid line, see Eq. (2))

model $[80,10]:$

$P_{n}(\phi) \approx \frac{1}{2 \pi}\left[1+a_{c}\left(3 \cos ^{2}\left(\phi-\phi_{c}\right)-1\right)\right]$,

where $a_{c}=0.3$ is an anisotropy parameter related to the fabric tensor [80]. The coefficient $\phi_{c} \approx 0.03 \mathrm{rad}$ is the most probable contact angle. As is the case here, it is usually collinear with the principal load direction.

2.2 Distribution of equivalent stresses and relation between discrete and continuum models

The forces in a granular model are point forces localized at the interface between grains. These quantities have no counterpart in continuum models. It is however possible to define generalizations of the notion of stress tensor to discrete media. Several proposals have been made in static $[28,88$, 66] and dynamic regimes [35,92], and we introduce here equivalent stresses for static (or quasi-static) loads. Given a network of contact forces $f_{\alpha}^{c}$ (at contact points $c$ and with coordinates $\alpha$ ) in a granular medium, the equivalent stress field $[58,111,66,102,108,72]$ is defined as

$\sigma_{\alpha \beta}^{V}=\frac{1}{V} \sum_{c=1}^{N_{c}} f_{\alpha}^{c} \ell_{\beta}^{c}=n_{c}\left\langle f_{\alpha} \ell_{\beta}\right\rangle=\sigma_{\beta \alpha}^{V}$,

where $\ell^{c}$ is the so-called branch vector, linking the centers of the two particles in contact at $c$, the sum in Eq. (3) is on the $N_{c}$ contact points in a cell with volume $V$, and $n_{c}=N_{c} / V$ is the density of contacts (see [111] for more details). Obviously, this quantity depends on the size of this cell volume (with more variability over the whole sample for smaller volumes). One of the main advantages of this definition is that it makes sense from the smallest scale (that of one contact point) to the largest one (that of the sample). It is also
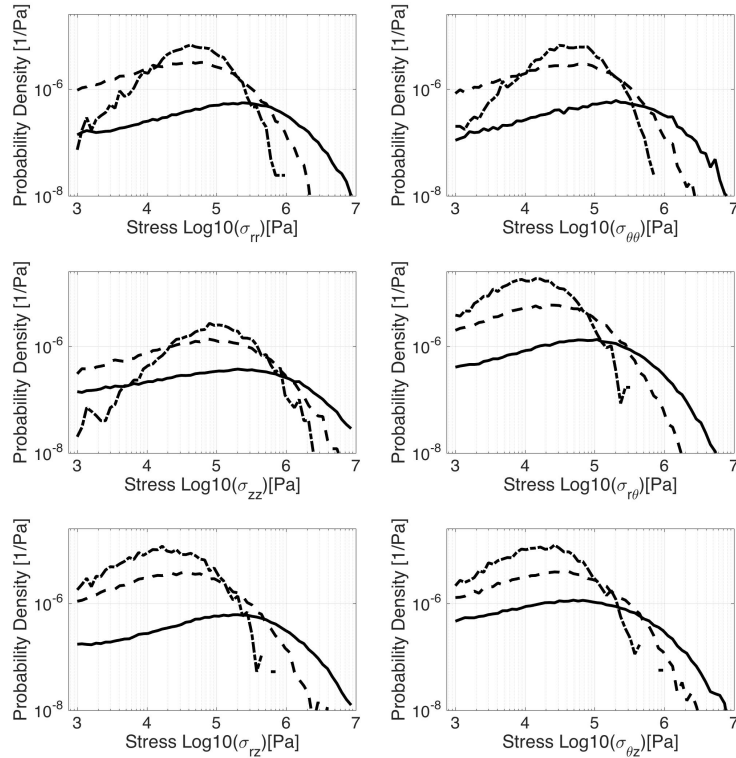

Fig. 4 Probability distribution function of the vertical component of the equivalent stress $\sigma_{z z}^{V}$, as a function of the averaging volume: $V=$ $(2 \mathrm{~cm})^{3}$ (solid line), $V=(4 \mathrm{~cm})^{3}$ (dashed line) and $V=(8 \mathrm{~cm})^{3}$ (dashdotted line).

an additive quantity, with respect to the volume (Lebesgue) measure.

Fig. 4 presents the probability distribution functions of the different components of the equivalent stress $\sigma^{V}$ (in a cylindrical basis) for different averaging volumes in a cube: $V=(2 \mathrm{~cm})^{3}=8 \mathrm{~cm}^{3}, V=(4 \mathrm{~cm})^{3}=64 \mathrm{~cm}^{3}$ and $V=$ $(8 \mathrm{~cm})^{3}=512 \mathrm{~cm}^{3}$. Due to the type of loading, the vertical component $\sigma_{z z}^{V}$ is obviously much larger than the other components. It is also clear that the distributions are wider (there is more variability) when the averaging volume is smaller. When averaging over larger volumes, a more homogeneous distribution is obtained, less variable and more clustered around the average value.

\section{Randomly-fluctuating heterogeneous continuum model of a granular medium}

In this section, we introduce the random model of Young's modulus. The random field is assumed stationary and ergodic, and we describe the choices that are made for the firstorder marginal law and correlation model. Most parameters are based on geometrical and mechanical information of the granular particles, obtained directly from the observation of discrete samples, and summarized in Section 3.1. The only exception is the variance, that is identified by solution of an inverse problem in Section 4. 


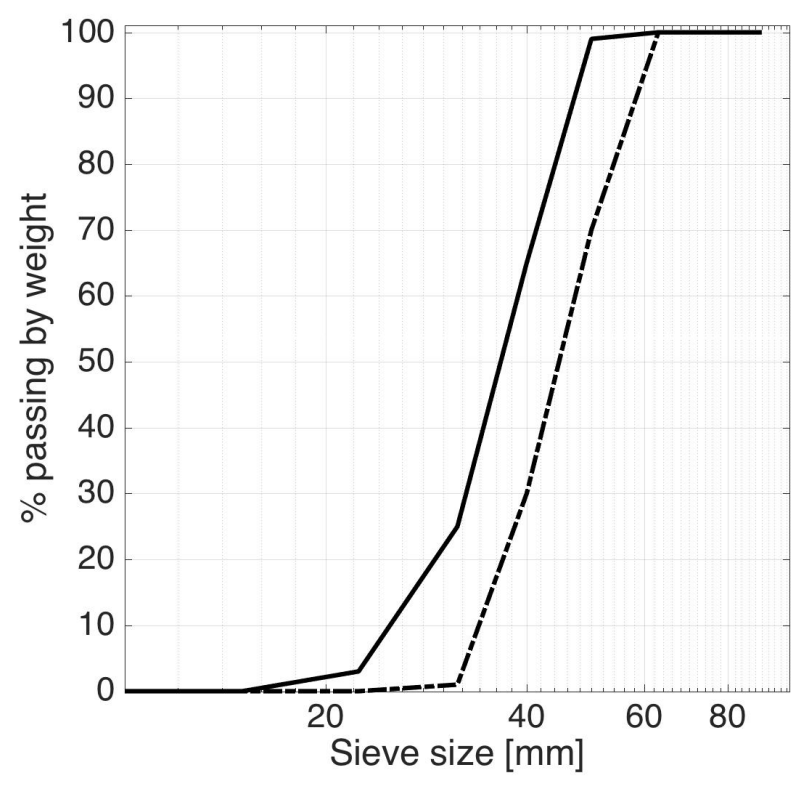

Fig. 5 Ballast gradation proposed by EN 13450 for railway ballast. The solid line represent the maximum limit, and the dashed line the minimum limit.

\subsection{Geometrical information on the granular packing}

The average diameter is defined as the mean (over all contacts and all samples) of the branch vector lengths:

$\underline{d}=\left\langle\frac{1}{N_{c}} \sum_{c=1}^{N_{c}}\left|\ell^{c}\right|\right\rangle$

where $\langle\cdot\rangle$ indicates an average over all discrete samples. In the set of cylindrical samples that we considered, we obtain $\underline{d} \approx 3.9 \mathrm{~cm}$. Alternatively, in the context of ballasted railway tracks or other engineering works, it would be possible to identify that average diameter directly from the normalized granulometry curves. Using for instance the minimum and maximum ballast gradations proposed by the standard EN 13450 (Fig. 5), one obtains average diameters between $\underline{d}=$ $3.6 \mathrm{~cm}$ and $\underline{d}=4.5 \mathrm{~cm}$.

On the same granular packings, we can also measure the packing density, defined as the volume of the grains relative to the total volume of the cylinder:

$\underline{\phi}=\left\langle\frac{V_{\text {grains }}}{4 \pi R^{2} H}\right\rangle$,

where $V_{\text {grains }}$ indicates the cumulated volume of all the grains in a given sample. Note that, as we will consider a continuum model with volume $4 \pi R^{2} H$ (that is to say with no holes), we will consider a density

$\rho=\underline{\phi} \rho_{\text {grains }}$ where $\rho_{\text {grains }}$ is the density of the constituent of the grains. This diminished density ensures that the total weight of the continuum samples will be the same as that of the discrete samples. It might be interesting to consider a fluctuating model of the density, but this will be investigated in a future work. In our model we use for density model: $\rho_{\text {grains }}=$ $2700 \mathrm{~kg} / \mathrm{m}^{3}, \underline{\phi}=0.5830$ and $\rho \approx 1574 \mathrm{~kg} / \mathrm{m}^{3}$.

\subsection{Correlation model for Young's modulus}

Considering the geometrical information above, a correlation model for the random field of Young's modulus is now chosen. In order to propose a theoretical correlation model, we assume in this section that the considered material is idealized as a two-phase medium: void matrix and impenetrable spheres. Even though the random field of Young's modulus corresponds to a unique phase, it is believed that proceeding this way will provide an appropriate order of magnitude of the scale of fluctuations in space. The amplitude of fluctuations (variance) will be identified in Section 4. We propose to consider the correlation structure of a dense packing of impenetrable spherical particles of diameter $\underline{d}$, with volume ratio $\phi$, developed by Torquato and coworkers $[104,103,79]$. This function depends only on $\phi$ and $\underline{d}$, and shows in particular the impenetrability condition and cosine decaying behavior, more pronounced with increased volume ratio. This theoretical model is based on the Meyer representation of the canonical function, by which the 2-point matrix probability function $S_{2}(r)$ can be written as a sum of a finite number of terms. Formally, this relation reads, using also the Ornstein-Zernike relation:

$S_{2}(r)=1-n V_{2}(r)+n^{2} \mathscr{F}^{-1}\left[\tilde{m}(k)^{2} \frac{\tilde{c}(k)}{1-n \tilde{c}(k)}\right]$,

where $\mathscr{F}^{-1}[\cdot]$ denotes the inverse Fourier transform of a function of the $k$ variable, $n$ (denoted $\rho$ in the original papers $[104,103,79])$ is the number density of the particles and $V_{2}(r)$ is the volume of the union of two spheres whose centers are separated by $r$, equal to

$V_{2}(r<\underline{d})=\frac{4 \pi}{3}\left(1+\frac{3}{4} \frac{2 r}{\underline{d}}-\frac{1}{16}\left(\frac{2 r}{\underline{d}}\right)^{3}\right)$

for $r<\underline{d}$ and $V_{2}(r>\underline{d})=8 \pi / 3$ otherwise. The Fourier transform of the indicator function of the particle (equal to 1 inside the particle, and 0 outside) $\tilde{m}(k)$ is equal to

$\tilde{m}(k)=\frac{4 \pi}{k}\left(\frac{\sin k}{k^{2}}-\frac{\cos k}{k}\right)$ 
and, using the Percus-Yevick approximation, the Fourier transform of the direct correlation function is given by:

$$
\begin{aligned}
& \tilde{c}(k)=-\frac{4 \pi}{k^{3}}\left\{\lambda_{1}[\sin (2 k)-2 k \cos (2 k)]\right. \\
& +\frac{3 \eta \lambda_{2}}{k}\left[4 k \sin (2 k)+\left(2-4 k^{2}\right) \cos (2 k)-2\right] \\
& +\frac{\eta \lambda_{1}}{2 k^{3}}\left[\left(-2 k^{4}+6 k^{2}-3\right) \cos (2 k)\right. \\
& \left.\left.+\left(4 k^{3}-6 k\right) \sin (2 k)+3\right]\right\},
\end{aligned}
$$

with $\eta=4 \pi n / 3, \lambda_{1}=(1+2 \eta)^{2} /(1-\eta)^{4}$ and $\lambda_{2}=-(1+$ $\eta / 2)^{2} /(1-\eta)^{4}$. Finally, the sought correlation model $\mathscr{R}(r)$, which is a normalized autocovariance, is directly related to the 2-point matrix probability function through the relation (see [103, Chapter 2]):

$\mathscr{R}(r)=\frac{S_{2}(r)-(1-\eta)^{2}}{\eta(1-\eta)}$

The reason for normalizing is to remove the discontinuity at the origin, in order to obtain a continuous model of Young's modulus. The variance will be identified in Section 4. Other, more complex, theories describe the correlation pattern for polydisperse packings of sphere $[109,15,16,36,25,52,103]$ and are expected to behave better at long distance, but have not been considered to date. The Meyer representation proposed by Quintanilla [79] for randomly oriented ellipsoids may be studied in future works. Theoretically, the PercusYevick approximation is not appropriate for dense arrays of particles, such as those that we are considering, but this approximation can be improved, in particular using the VerletWeis [107] or Kincaid-Weis [49] semi-empirical modifications to the Percus-Yevick radial distribution. In this work, we considered and used the modifications proposed by VerletWeis. A correction is therefore applied on the diameter and on the coefficient $\eta$. The corrected value of the diameter is given by $\frac{d_{w}{ }^{3}}{} / \eta_{w}=d^{3} / \eta=6 /(\pi n)$, where $\eta_{w}=\eta-\eta^{2} / 16$, and $\eta_{w}$ is the corrected coefficient $\eta$. These corrections replace the original values in the formulas. This empirical correction tries to reduce, for large $r$, the main maximum of the structure factor, and the small difference in the phase in the radial distribution function. Finally, the correlation model of Eq. (11) is plotted in Fig. 6, for different volume fractions and diameters.

\subsection{First-order marginal law for Young's modulus}

We now turn to the choice of the first-order marginal law. Young's modulus is a positive quantity, and the choice of first-order marginal law should reflect that physical requirement. We consider in this paper two of these: (i) log-normal law, and (ii) Gamma law. Both models are parameterized

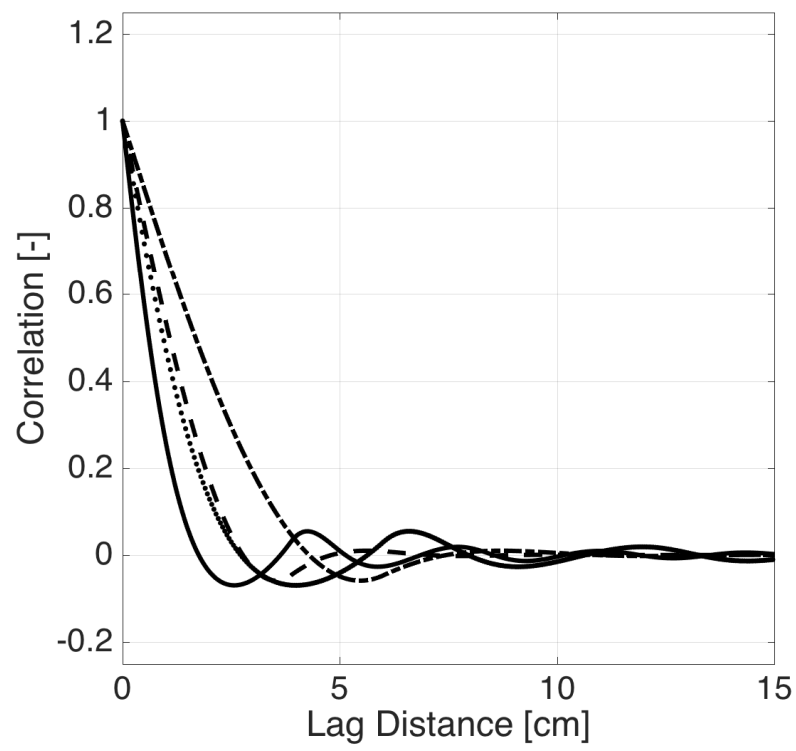

Fig. 6 Correlation function for an impenetrable sphere packing with constant diameter $\underline{d}$ and volume ratio $\phi$. Four cases were presented here: $\underline{d}=3.9 \mathrm{~cm}$ and $\phi=0.5682$ (solid line); $\underline{d}=3.9 \mathrm{~cm}$ and $\phi=0.2$ (dashed line); $\underline{d}=6 \overline{\mathrm{cm}}$ and $\phi=0.5682$ (dotted line); $\underline{d}=6 \overline{\mathrm{cm}}$ and $\underline{\phi}=0.2$ (dash-dotted line).

by an average $\mu_{E}$ and variance $\sigma_{E}^{2}$. The probability density function of the log-normal distribution is:

$\mathbb{P}(E)=\frac{1}{E s \sqrt{2 \pi}} \exp \left(-\frac{(\ln E-m)^{2}}{2 s^{2}}\right)$

where $s^{2}=\ln \left(1+\sigma_{E}^{2} / \mu_{E}^{2}\right)$ and $m=\ln \left(\mu_{E}\right)-s^{2} / 2$ are the variance and mean of the underlying Gaussian distribution. The probability densify function of the Gamma distribution is:

$\mathbb{P}(E)=\frac{E^{k-1}}{\Gamma(k) \theta^{k}} \exp \left(\frac{-E}{\theta}\right)$,

where $\theta=\sigma_{E}^{2} / \mu_{E}$ and $k=\mu_{E}^{2} / \sigma_{E}^{2}$ are the shape parameters of the Gamma distribution, and $\Gamma(k)=\int_{0}^{+\infty} t^{k-1} \exp (-t) d t$ is the Gamma function. An example of these two densities (for $\mu_{E}=1 \mathrm{~N} / \mathrm{m}^{2}$ and $\sigma_{E}^{2}=10 \mu_{E}^{2}$ ) is plotted in Fig. 7. They mainly differ in the tails, that is to say for the description of very small and very large values. Note also that, as desired for a positive parameter, the support of both functions is limited to $\mathbb{R}^{+}$.

3.4 Generation of realizations of the stochastic field of Young's modulus

As realizations of the stochastic field of Young's modulus will be required for the numerical simulations in the next 


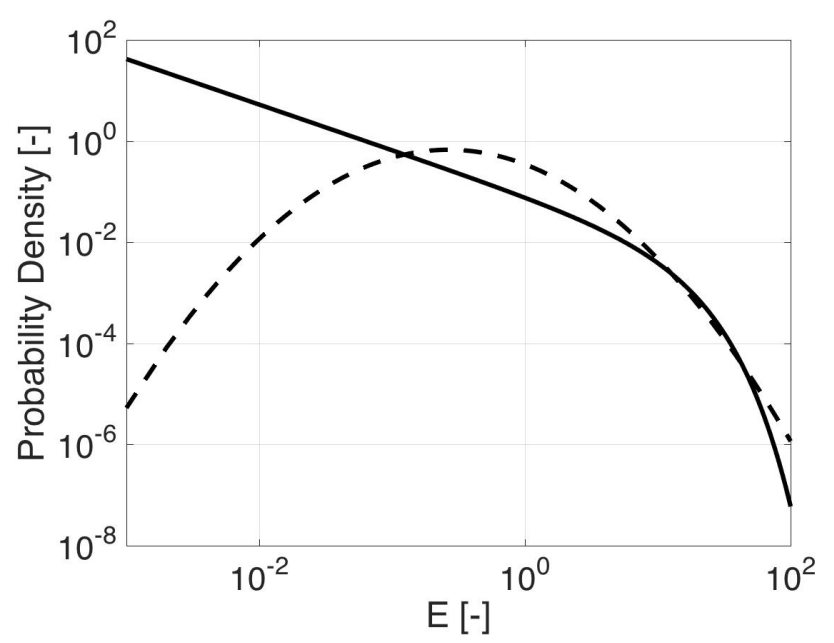

Fig. 7 Probability density function for the Gamma distribution (solid line) and log-normal distribution (dashed line) for $\mu_{E}=1 \mathrm{~N} / \mathrm{m}^{2}$ and $\sigma_{E}^{2}=10 \mu_{E}^{2}$.

sections (cylindrical samples in Section 4 and a ballasted railway track in Section 5), we conclude this section with a description of a numerical strategy for the generation of samples. This can be performed in two different ways: either in the space or in the spectral domain. The latter is widely used for its simplicity and efficiency [95]. It consists in summing a large number of functions oscillating with random phases and with amplitudes designed to match the desired correlation model:

$E(x)=2 \sum_{j=1}^{N} \sqrt{\sigma_{E}^{2} \hat{\mathscr{R}}\left(k_{j}\right) \Delta k} \zeta_{j} \cos \left(k_{j} x+\phi_{j}\right)$

where the $\zeta_{j}$ are independent unit centered gaussian random variables, the $\phi_{j}$ are independent random variables, uniform over $[0,2 \pi], N$ in the number of terms in the sum, $\Delta k$ is the discretization step in wave-number space, and $\hat{\mathscr{R}}\left(k_{j}\right)$ is the normalized power spectral density function in wave-number space, that is to say the Fourier transform of the normalized autocovariance introduced in Eq. (11). Although written here for simplicity in $1 \mathrm{D}$, the extension to $3 \mathrm{D}$ is described in full details in [96]. This algorithm generates random fields that are asymptotically Gaussian and asymptotically ergodic, so that a Rosenblatt transformation [87] is then applied to obtain the desired first-order marginal density. Note that this pointwise transformation is known to modify in general the correlation structure $[37,78]$.

The efficiency of this technique mainly lies in the possibility to take advantage of the efficiency of the Fast Fourier Transform (FFT) algorithm in a straightforward way [95, 96]. However, when simulating realizations over large clusters of computers, as required in the simulation in Section 5, the algorithmic complexity of the FFT is too large. We therefore consider a particular implementation of the spectral representation method, which considers independent realiza-

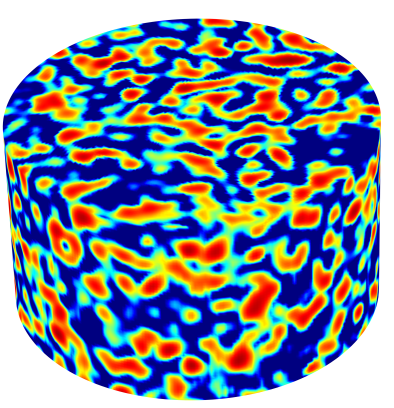

Fig. 8 One realization of the random model of Young's modulus for a cylinder of the same size as in Fig. 1: radius $R=35 \mathrm{~cm}$ and height $H=39 \mathrm{~cm}$.

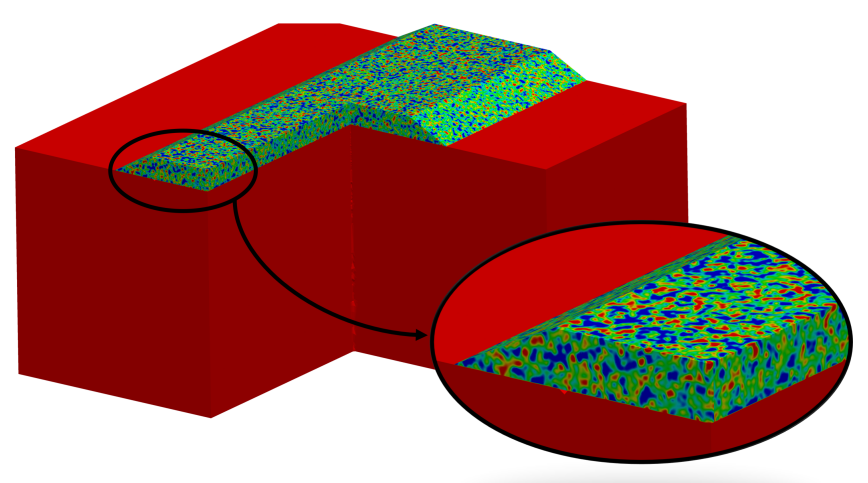

Fig. 9 One realization of the random model of Young's modulus for a portion of ballasted track, with the same size as in Fig. 21: height $H=48 \mathrm{~cm}$, width between $L_{b}=5.00 \mathrm{~m}$ at the bottom and $L_{b}=3.90 \mathrm{~m}$ at the top.

tions (obtained with the scheme above) over each processor and overlap and merging to retrieve the continuity and correlation over the global domain. More details on this implementation can be found in [75]. Two examples of realizations of random fields are proposed: a typical sample used in Section 4 is presented in Fig. 8 and a typical sample used in Section 5 is presented in Fig. 9. Finally, in Fig. 10, a comparison is proposed between the target correlation structure and the correlation structure estimated from one single realization of the Rosenblatt-transformed random field (generated for the cylinder of Section 4 and Fig. 8). As expected, a slight shift in the correlation length seems to be observed, but the shape remains quite unchanged. The influence of the correlation structure of the random field is discussed in more details in Section 4.5.

\section{Identification of the continuum model parameters}

This section discusses the identification of the variance of the stochastic model of Young's modulus described in the previous section. The reference for the identification is the 


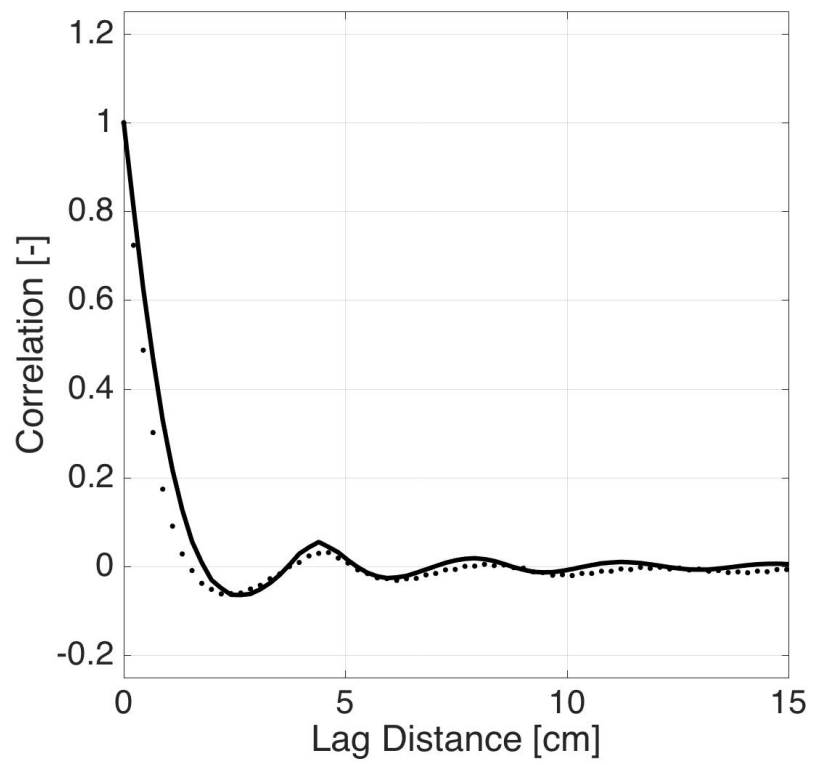

Fig. 10 Comparison of the theoretical correlation model, used to generate the Gaussian random fields (solid line) and the correlation estimated from one cylindrical sample of the Rosenblatt-transformed random field (dotted line).

distribution of equivalent stresses $p_{r}\left(\sigma_{z z}\right)$ in the set of numerical granular samples described in Section 2.

\subsection{General methodology}

\subsubsection{Identification process}

The steps of the identification process are summarized in Fig. 11 and listed below:

1. an initial value of the variance is chosen

2. a realization of a heterogeneous Young's modulus field is generated

3. the distribution of stresses $p\left(\sigma_{z z}\right)$ in the Finite Element model (described in Section 4.1.2 below) is computed

4. The $\mathrm{L}^{2}$ distance between that distribution and the reference is computed

$L_{r}=\int_{\Sigma}\left(p\left(\sigma_{z z}\right)-p_{r}\left(\sigma_{z z}\right)\right)^{2} d \sigma_{z z}$

The interval $\Sigma \in \mathbb{R}$ is chosen so as to remove the tails of the experimental distribution, for which the data is too scarce to ensure statistical convergence. In this work, we have considered the interval $\left[1 \times 10^{4}-7 \times 10^{6}\right] \mathrm{N} / \mathrm{m}^{2}$, which corresponds to $90 \%$ of the experimental probability density of $\sigma_{z z}$.

5. Depending on the distance obtained, and using a simplex algorithm [53], new values of the variance are proposed, and an iterative process is considered on steps 1-5.

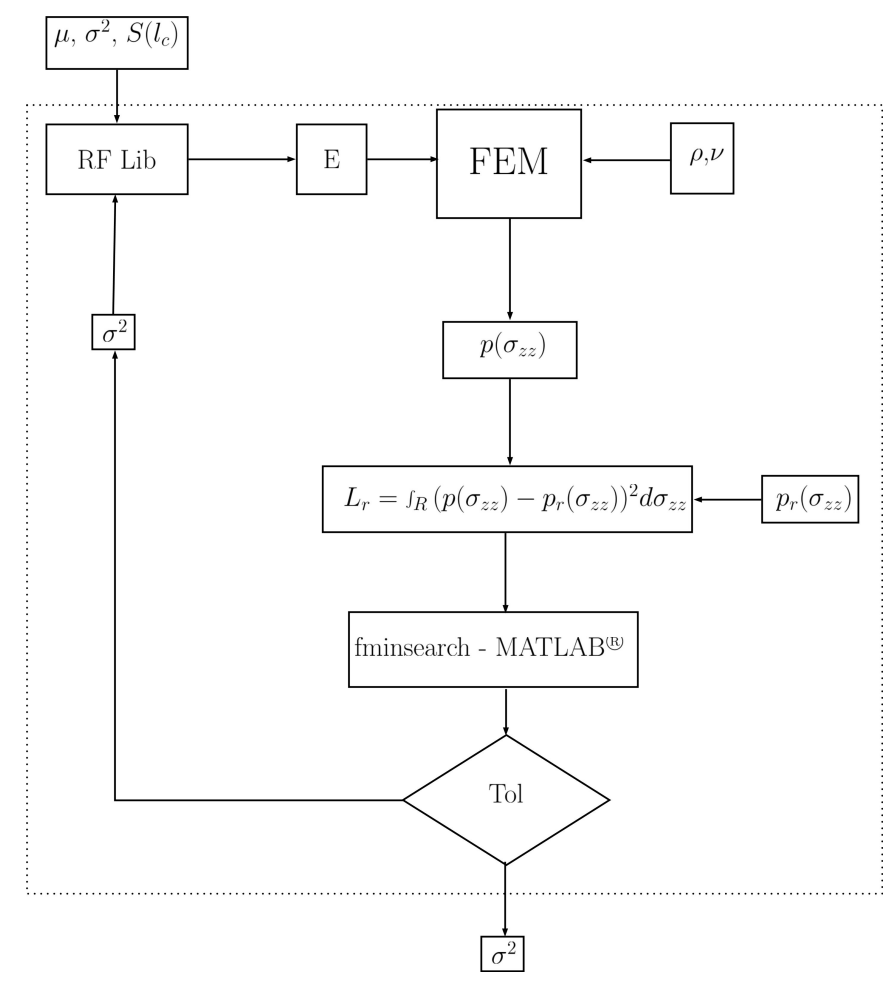

Fig. 11 Optimization scheme.

6. If convergence is not obtained, a new value of the variance is proposed and a new iteration starts at step 2; else the algorithm exits.

Note that in the entire process, only the variance evolves. The remaining parameters (average Young's modulus, correlation length and model, first-order marginal density), as well as the averaging volume for the reference distribution, are fixed. Note also that the Kullback-Leibler distance [8] has been tested instead of Eq. (15) and yielded the same qualitative results as those presented below.

\subsubsection{Description of the continuum samples used for identification}

The continuum samples that are used for the identification process imitate the discrete samples described in Section 2. They are cylinders of radius $R=35 \mathrm{~cm}$ and height $H=39 \mathrm{~cm}$, loaded with an isotropic confinement pressure of $60 \mathrm{kPa}$ on the lateral face and with a vertical pressure of $63 \mathrm{kN}$ on the upper face. Gravity is also considered within the model. The generation of one realization of the random field and the simulation the corresponding response took on average 4800 s of CPU time.

We consider a homogeneous density $\rho=1574 \mathrm{~kg} / \mathrm{m}^{3}$ (see Section 3.1) and a homogeneous Poisson ratio $v=0.23$ [45]. As the identification process is driven by stress distribution, and because we are considering a linear model, the average Young's modulus is expected to be ill-constrained 


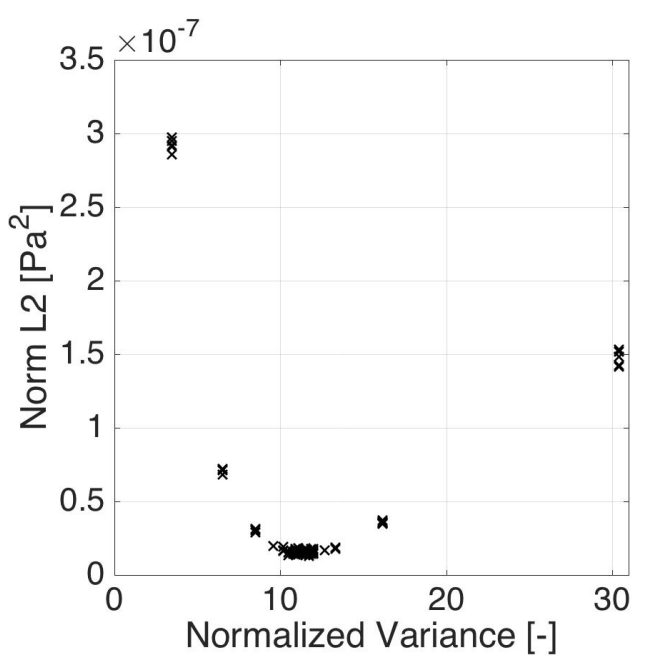

Fig. 12 Evolution of the $L^{2}$ distance during the optimization process for a Gamma first-order marginal distribution and averaging volume $V=(4 \mathrm{~cm})^{3}$ as a function of the normalized variance $\sigma_{E}^{2} / \underline{E}^{2}$.

in this experiment. We therefore choose to fix its value at $\underline{E}=80 \mathrm{MPa}[45]$, and will investigate this further in Section 4.6.

All simulations are performed with the Finite Element Method (COMSOL software) with a mesh of close to 380'000 linear hexahedra, corresponding to elements of size $h \approx 0.7 \mathrm{~cm}$, much smaller than the correlation length. To simplify the posttreatment process, note that output stresses are extracted only within a box of size $48 \times 48 \times 32(\mathrm{~cm})^{3}$, inset into the cylinders. It was checked that there is no significant difference between the stress distribution within the box and within the full cylinder.

4.2 Identification of a Gamma model for averaging volume $V=(4 \mathrm{~cm})^{3}$

Considering a Gamma first-order marginal density, and the experimental distribution of equivalent stresses obtained with an averaging volume of $V=(4 \mathrm{~cm})^{3}$, the optimization process leads to the evolution of the $\mathrm{L}^{2}$ distance plotted in Fig. 12. Although there is variability between samples, there is clearly a minimum of the $\mathrm{L}^{2}$ distance, with a normalized variance close to $\sigma_{E}^{2} / \underline{E}^{2} \approx 11$. Note that the minimization was restarted several times to make sure that no bias resulted from sample-to-sample variability. In each restart the departure point changed.

The distributions of continuum stresses are plotted in Fig. 13 (solid lines) and compared to the experimental equivalent stresses (dashed lines). The identified stress distributions match very well with the discrete ones, both for the $\sigma_{z z}$ coefficient, which was used to drive the identification, and the other coefficients, that were not used in the identification. The only apparent deficiency of the model lies
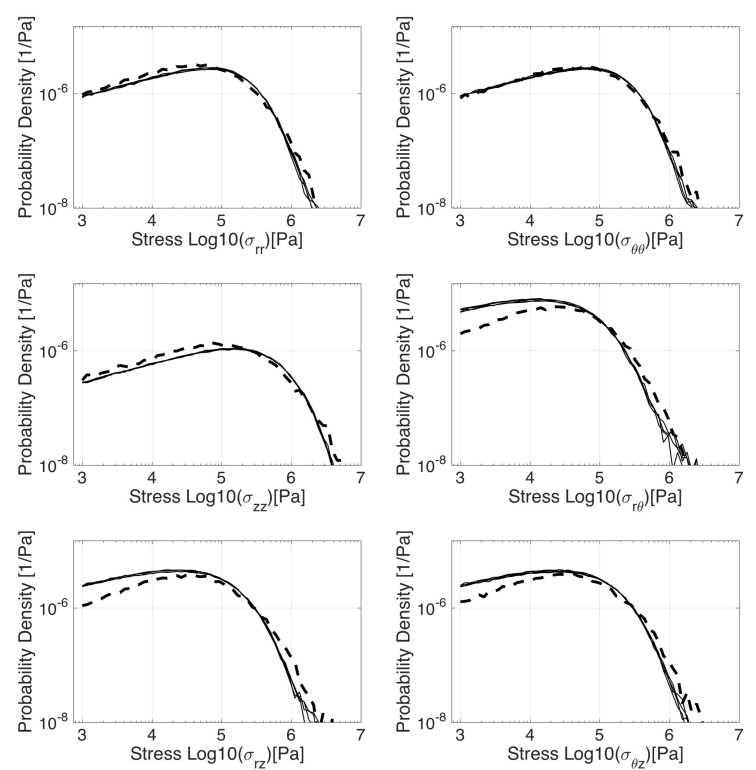

Fig. 13 Distribution of the logarithm of the equivalent stresses for the discrete samples and averaging volume $V=(4 \mathrm{~cm})^{3}$ (dashed line), and for the identified continuum model with Gamma first-order marginal density (solid line).

in a slightly higher value of the lower-than-average shear stresses in the identified continuum model with respect to the experimental ones. Note that, on each plot, there are actually several continuous lines, corresponding to different realizations of cylindrical samples with the same statistical parameters. This shows that a cylindrical sample is statistically representative for the distribution of stresses, because there is almost no variability from sample to sample.

\subsection{Identification of Gamma models for different averaging} volumes

Still considering the Gamma first-order marginal density, we now consider a different averaging volume $V=(8 \mathrm{~cm})^{3}$ and compare it to the previous volume $V=(4 \mathrm{~cm})^{3}$. The optimization process produced the results plotted in Fig. 14. A clear reduction of the normalized variance can be observed, from $\sigma_{E}^{2} / \underline{E}^{2} \approx 11$ for $V=(4 \mathrm{~cm})^{3} \sigma_{E}^{2} / \underline{E}^{2} \approx 4$ for $V=(8 \mathrm{~cm})^{3}$ compared to the variance for $V=(4 \mathrm{~cm})^{3}$. Note that we do not consider here the identification for the averaging volume $V=(2 \mathrm{~cm})^{3}$ because the generation of random fields with Gamma first-order marginal densities and normalized variances larger than 26 resulted in numerical issues. This is linked to the inversion of the Gamma cumulative function in the Rosenblatt transform and will be investigated later.

The obtained distributions of identified continuum stresses $\left(\sigma_{z z}\right.$ component) are plotted in Fig. 15 and compared to the 


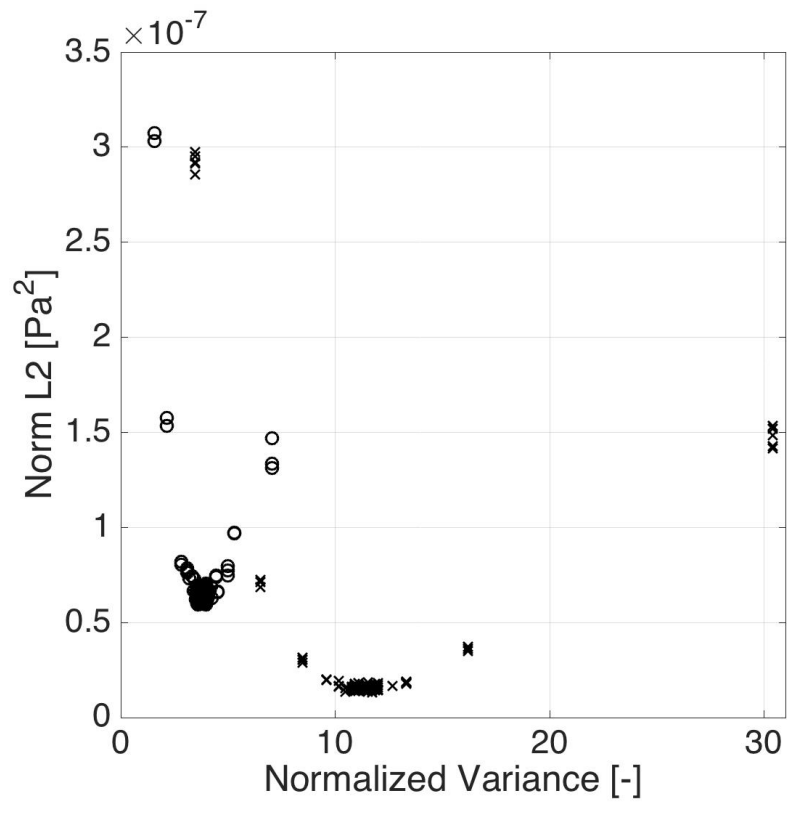

Fig. 14 Evolution of the $L^{2}$ distance during the optimization process for a Gamma first-order marginal distribution and different averaging volumes: $V=(4 \mathrm{~cm})^{3}$ (crosses), and $V=(8 \mathrm{~cm})^{3}$ (circles), as a function of the normalized variance $\sigma_{E}^{2} / \underline{E}^{2}$.

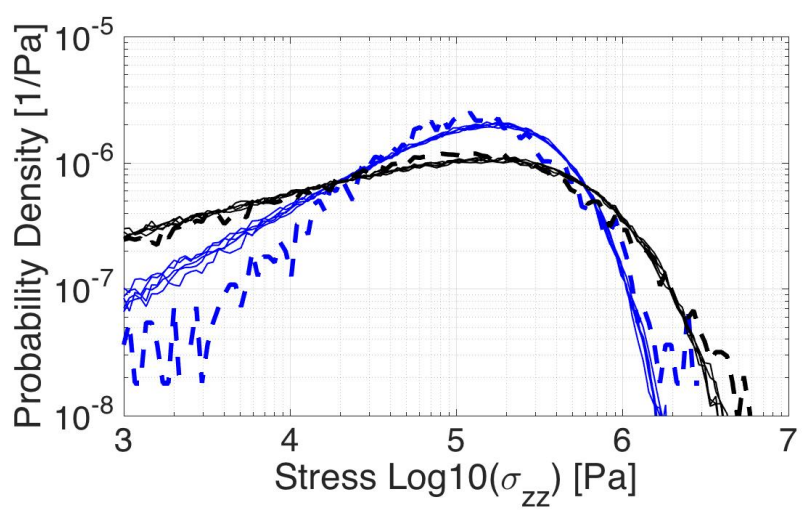

Fig. 15 Distribution of the logarithm of the vertical equivalent stresses, $\sigma_{z z}$ for the discrete samples (dashed lines) and for the identified continuum models with Gamma first-order marginal density (solid lines), for averaging volumes $V=(4 \mathrm{~cm})^{3}$ (black lines) and $V=(8 \mathrm{~cm})^{3}$ (blue lines).

experimental equivalent stresses. The variance reduction is clearly visible. The agreement between both groups of curves is very good, although less for the larger average volume, where the lower-than-averages forces are slightly overestimated. However, it should be noted that they correspond to very low probability of occurence (two orders of magnitude below the maximum), so that a larger experimental database might be desirable.

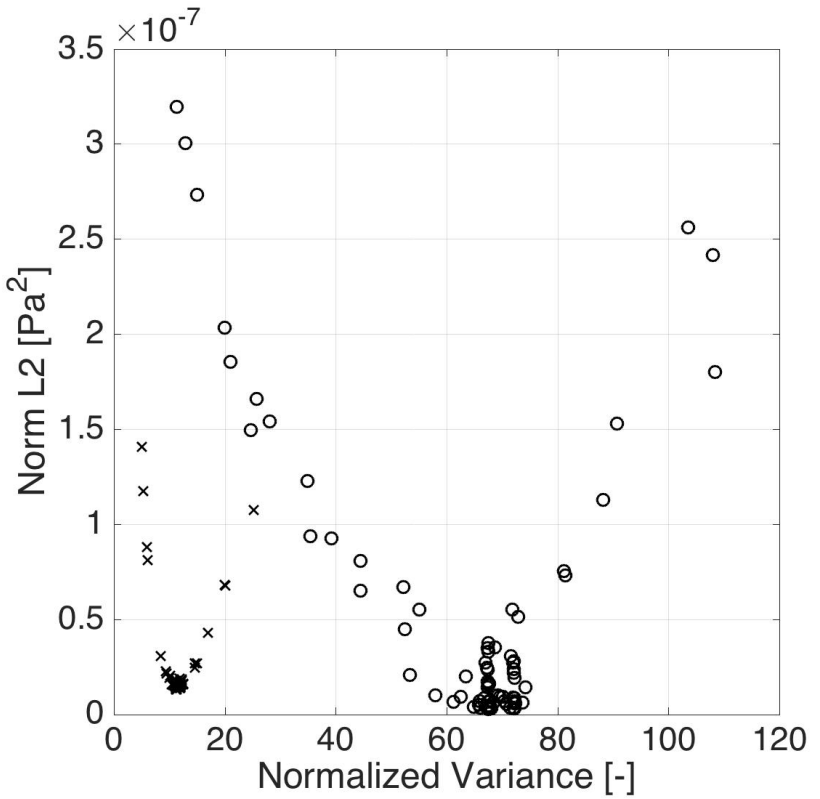

Fig. 16 Evolution of the $L^{2}$ distance during the optimization process at $V=(4 \mathrm{~cm})^{3}$ as a function of the normalized variance $\sigma_{E}^{2} / \underline{E}^{2}$, for a Gamma (crosses) and log-normal (circles) first-order marginal distribution.

\subsection{Influence of the first-order marginal density}

Considering the averaging volume of $V=(4 \mathrm{~cm})^{3}$, we now consider a log-normal first-order marginal density, and compare the results obtained with those of the Gamma firstorder marginal density (Section 4.2). The optimization process leads to the convergence presented in Fig. 16. It can be seen that the variance corresponding to the log-normal density is much larger than before: $\sigma_{E}^{2} / \underline{E}^{2} \approx 72$. It is also interesting to remark that there is much more variability in the $\mathrm{L}^{2}$ distance depending on the particular realization of cylindrical sample that is considered.

The stress distributions are then plotted in Fig. 17. Although the distribution for the $\sigma_{z z}$ coefficient matches relatively well the experimental equivalent stress, as expected out of the optimization process, all the other coefficient are rather badly approximated. This is probably due to the very large difference between the log-normal and Gamma distributions for the lower-than-average values (see Fig. 7). The log-normal first-order marginal density can therefore not be considered as appropriate to model Young's modulus in the ballast.

\subsection{Influence of the correlation model}

To consider the influence of the correlation model in the identification process, we compared the model presented in Sec. 3.2 with a cardinal sine correlation model $[97,100]$. The 

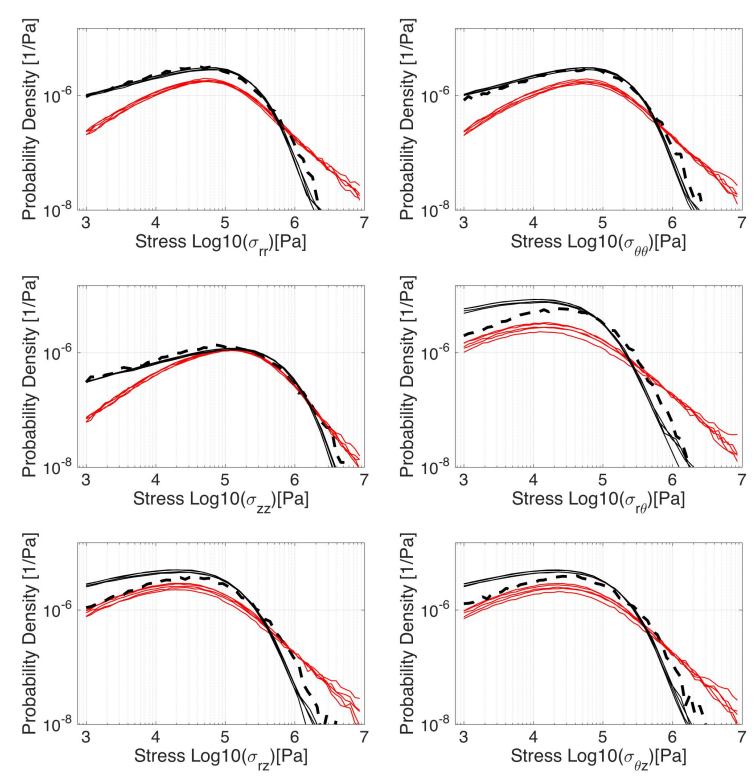

Fig. 17 Distribution of the logarithm of the equivalent stresses for the discrete samples and averaging volume $V=(4 \mathrm{~cm})^{3}$ (black dashed line), for the identified continuum model with Gamma first-order marginal density (black solid line), and for the identified continuum model with Log-normal first-order marginal density (red solid line).

optimization process leads to the convergence presented in Fig. 18. A remarkable influence of the departure point on the values at convergence was observed. Indeed, when the departure point was lower than about $10\left(\sigma_{E}^{2} / \underline{E}^{2} \approx 10\right)$, the results were quite consistent, and the minimum was found close to $\sigma_{E}^{2} / \underline{E}^{2} \approx 5$. However, when the initial point was larger than 10 , the convergence process became stuck in local minima (see the square markers in Fig. 18). Note that the normalized variance found in this case is quite different from the value found in Sec. 4.2 for the same volume and first-order marginal law.

The stress distributions are then plotted in Fig. 19. The distribution for the $\sigma_{z z}$ around and above the mean value is quite satisfactory, except for one realization of the optimization process. The lower-than-average stresses are however poorly represented. For all other components of the stress tensor, both the below-than-average and above-average stresses are badly represented. Finally, note that the realization with a departure point larger than 10 presents stress distributions whose shapes are completely different from the granular ones.

\subsection{Influence of the average of Young's modulus}

Finally, we consider a different average Young's modulus and compare the results to the case in Section 4.2. Fig. 20 presents the convergence of the $\mathrm{L}^{2}$ distance for $\underline{E}=80 \mathrm{MPa}$

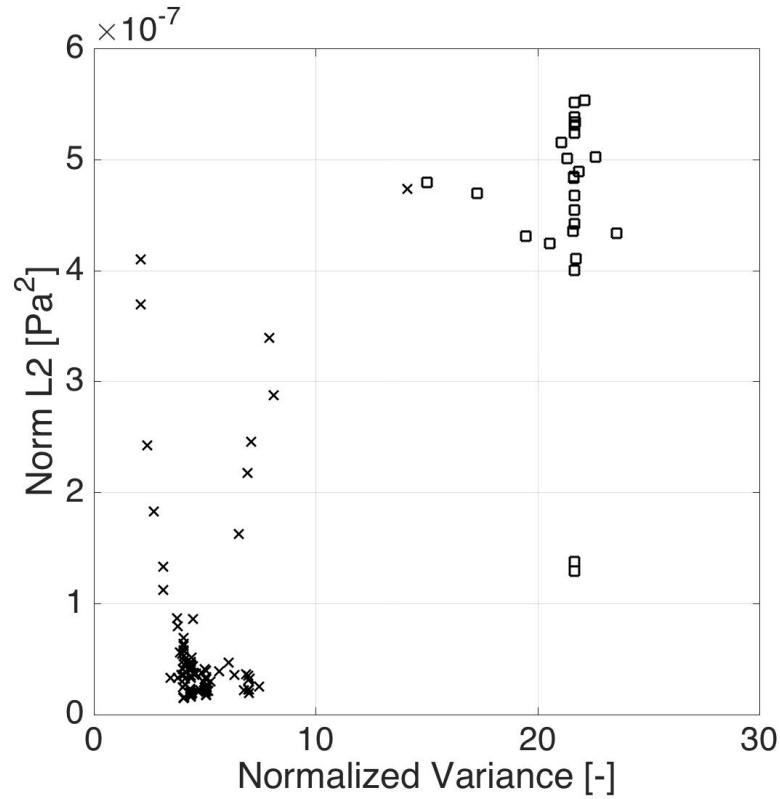

Fig. 18 Evolution of the $L^{2}$ distance during the optimization process at $V=(4 \mathrm{~cm})^{3}$ as a function of the normalized variance $\sigma_{E}^{2} / \underline{E}^{2}$. The crosses represents the realizations where the departure point, for the optimization process, were below $10, \sigma_{E}^{2} / \underline{E}^{2} \leq 10$. The square marker represent the realization where the departure point was greater than 10 , $\sigma_{E}^{2} / \underline{E}^{2} \geq 10$.
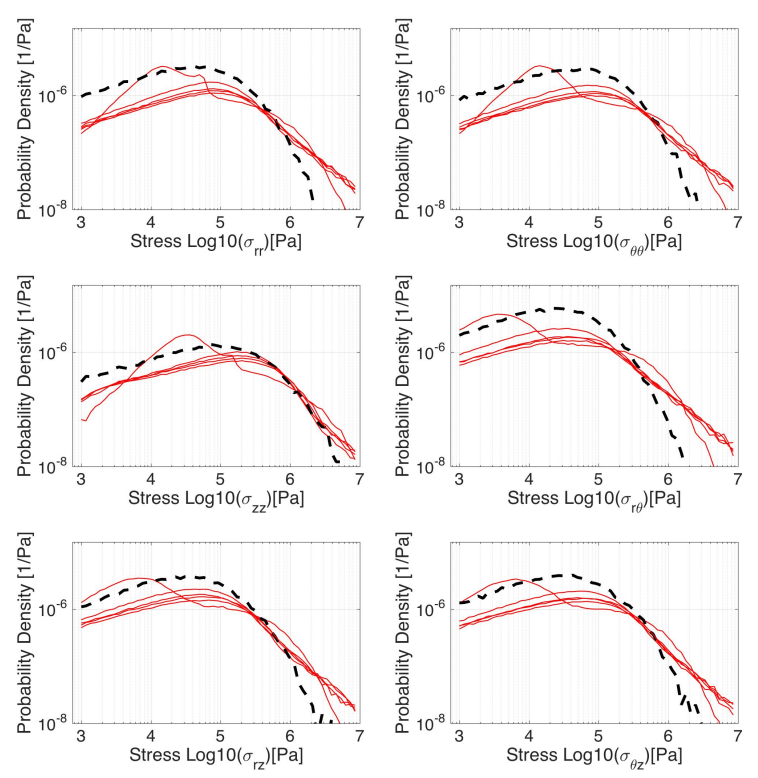

Fig. 19 Distribution of the logarithm of the equivalent stresses for the discrete samples and averaging volume $V=(4 \mathrm{~cm})^{3}$ (black dashed line), for the identified continuum models with Gamma first-order marginal density and cardinal sine correlation model (red solid lines).

(with crosses) and $\underline{E}=800 \mathrm{MPa}$ (with circles). It is clear from the orders of magnitude of the $\mathrm{L}^{2}$ distance (compared 


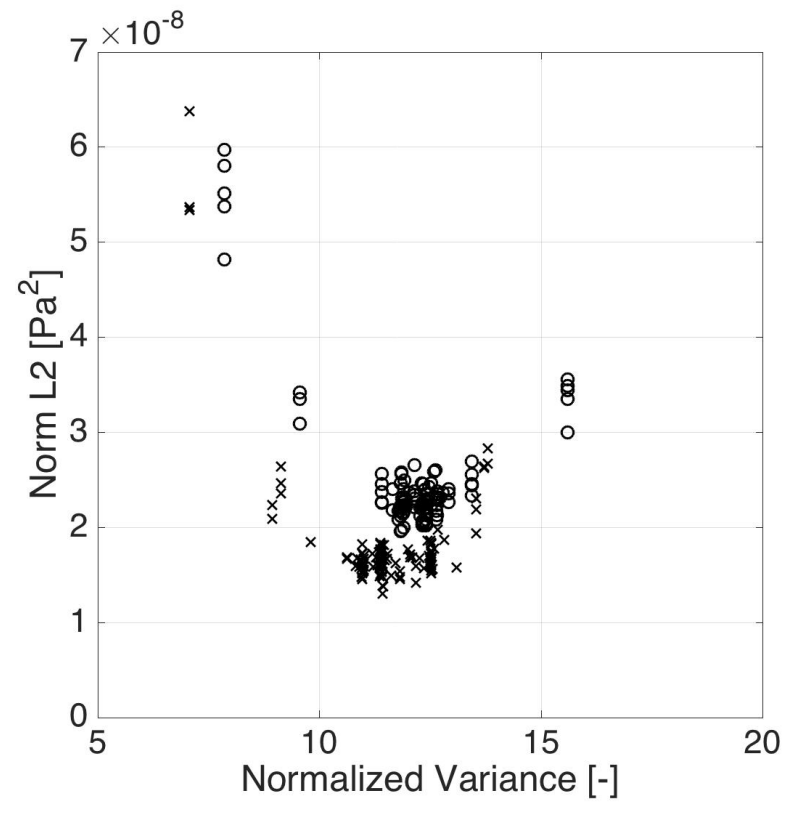

Fig. 20 Evolution of the $L^{2}$ distance during the optimization process for a Gamma first-order marginal distribution at $V=(4 \mathrm{~cm})^{3}$ and $\underline{E}=$ $80 \mathrm{MPa}$ (crosses), and for $V=(4 \mathrm{~cm})^{3}$ and $\underline{E}=800 \mathrm{MPa}$ (circles), as a function of the normalized variance $\sigma_{E}^{2} / \underline{E}^{2}$.

to the previous figures) that the average Young's modulus has very little influence on the identification. This was to be expected because the identification is performed on stress distributions rather than on kinematical quantities. The stress distributions are not plotted because they almost overlap in the two cases.

As a general conclusion to this section, it seems that modeling the Young's modulus as a random field with Gamma first-order marginal density and granular correlation model allows to reproduce very convincingly the stress distribution in a granular sample. The variance depends on the averaging volume for the equivalent stresses and has been identified as close to $\sigma_{E}^{2} / \underline{E}^{2} \approx 11$ for $V=(4 \mathrm{~cm})^{3}$. As expected, the average is not well constrained by the chosen experimental data.

\section{Application to the modeling of the ballast}

In this section, we consider the stochastic model of Young's modulus developed in the previous section and analyze the impact of the heterogeneity it models on a dynamic simulation of the passage of a train on a ballasted railway track. As discussed in the introduction, this simulation is not realistic because there is no dissipation involved. However, we believe it illustrates very clearly the importance of considering heterogeneity in the numerical modeling of granular media.

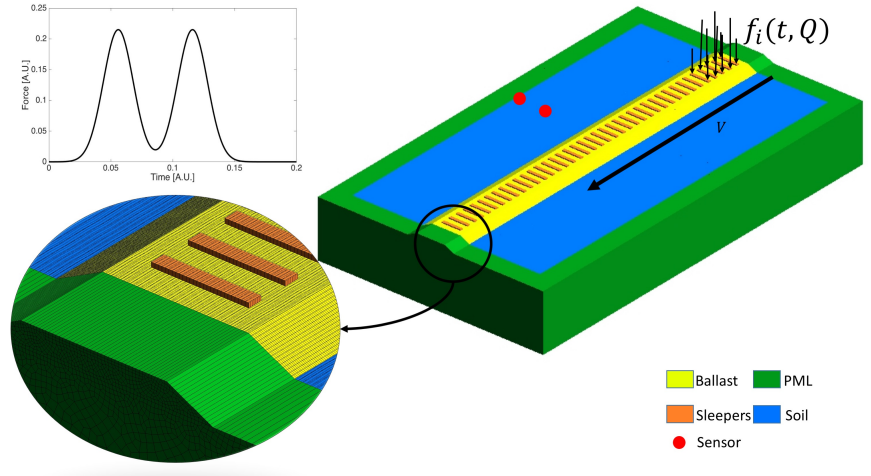

Fig. 21 Geometry, loading, boundary conditions and mesh for the wave propagation analysis.

\subsection{Description of the numerical model of the ballast}

We consider the model depicted in Fig. 21. It is a 38 m-long one-way track segment, with a ballast (in yellow on Fig. 21) of height $48 \mathrm{~cm}$ and width between $3.9 \mathrm{~m}$ at the top and $5 \mathrm{~m}$ at the bottom. Below the track, the soil is numerically modeled on a width of $20 \mathrm{~m}$ and a depth of $5 \mathrm{~m}$. The concrete sleepers have dimensions $20 \times 30 \times 200 \mathrm{~cm}^{3}$, are separated by $d=0.6 \mathrm{~m}$, and are embedded in the soil on a height of $10 \mathrm{~cm}$. This simple setup is representative of a realistic ballasted railway track.

In the simulated domain, the wave field is the solution of the wave equation:

$\nabla \cdot \sigma-\rho \ddot{\mathbf{u}}=\mathbf{0}$

where the material is assumed isotropic and linear, so that the strain is $\varepsilon=\left(\nabla \mathbf{u}+\nabla \mathbf{u}^{T}\right) / 2$ and the stress is $\sigma=\lambda(\mathbf{x}) \operatorname{Tr} \varepsilon \mathbb{I}+$ $2 \mu \varepsilon$, with $\lambda$ and $\mu$ the Lamé parameters, and $\mathbb{I}$ is the identity second-order tensor. The density is denoted $\rho$. The Lamé parameters are related to the wave velocities through $V_{p}=$ $\sqrt{(\lambda+2 \mu) / \rho}$ and $V_{s}=\sqrt{\mu / \rho}$. The soil is assumed homogeneous in all simulations, with $V_{s}=180 \mathrm{~m} / \mathrm{s}, V_{p}=350 \mathrm{~m} / \mathrm{s}$, and $\rho=1900 \mathrm{~kg} / \mathrm{m}^{3}$. The concrete sleepers are assumed homogeneous in all simulations, with $V_{s}=2500 \mathrm{~m} / \mathrm{s}, V_{p}=$ $4500 \mathrm{~m} / \mathrm{s}$, and $\rho=2400 \mathrm{~kg} / \mathrm{m}^{3}$. In the simulations where the ballast is assumed homogeneous, we consider $V_{s}=150 \mathrm{~m} / \mathrm{s}$, $V_{p}=380 \mathrm{~m} / \mathrm{s}$, and $\rho=1900 \mathrm{~kg} / \mathrm{m}^{3}$. In the simulations where the ballast is assumed heterogeneous, the random model of mechanical properties is the one described in the previous section, and the average values are taken equal to those of the homogeneous case.

The vertical loading considered is a classical one for modeling the influence of a train-rail system (one bogie) on the sleepers [38]. It considers the flexibility of the rail by transferring the point loads of the boggie of a train onto consecutive sleepers. The movement of the train is taken into account by moving the position of the point loads at the appropriate velocity. The loading on each of the sleepers (and 
on each side of each sleepers, as indicated on Fig. 21) is given by:

$F_{i}(t)=\frac{Q Y}{2}\left[C^{\frac{\left(v_{0}\left(t-\delta_{i}\right)-a\right)^{2}}{d^{2}}}+C^{\frac{\left(v_{0}\left(t-\delta_{i}\right)-a-L\right)^{2}}{d^{2}}}\right]$

where $Q$ is the load magnitude, $L=3 \mathrm{~m}$ is the wheelbase, $a=5 d=3 \mathrm{~m}$ is the critical distance, beyond which the load is assumed to vanish, $v_{0}=100 \mathrm{~m} / \mathrm{s}$ is the chosen train velocity, and $C=0.61$ and $Y=0.41$ are constants that depend of the soil-ballast combination. The latter values are obtained from the experimental values in [4]. In order to produce a "moving load" each sleeper is associated with a delay $\delta_{i}$. The Fig. 21 highlights the region where the vertical point loads are applied at the top of the sleepers at the initial time. The transmission of the point load to the ballast layer takes place through the sleepers. Only five sleepers are loaded in the image because the influence of the boggie on further sleepers almost vanishes.

In the inset of Fig. 21, the refinement of the mesh is also shown. The mesh is discretized with 0.81 millions of hexahedral elements. High-order polynomials of order 7 are used in the spectral element solver (see Section 5.2), which corresponds to 343 degrees of freedoms (DOFs) for each element, and a total of close to 175 millions of DOFs for the entire mesh. On the exterior of the soil box (green area in Fig. 21), a Perfectly Matched Layer [32] is added to absorb outgoing waves.

\subsection{Description of the solver}

To approximate the solution of the wave equation, the solver used in this paper is based on the Spectral Element Method (SEM, see [21] for mathematical details and [50] for an example in seismology). The SEM is a high-order FEM that uses a Gauss-Lobatto-Legendre quadrature rule for integration and Lagrange polynomials based on the nodes of that quadrature. Inserting the polynomial functions and quadrature rules into the variational form of Eq. (16) leads to a system of ordinary differential equations:

$\mathbf{M V}=\mathbf{F}_{\text {ext }}-\mathbf{F}_{\text {int }}(\mathbf{U})$

where $\mathbf{U}$ and $\mathbf{V}$ are vectors containing the components of the displacement and velocity at the nodes, respectively, $\mathbf{M}$ is the mass matrix, and the vectors $\mathbf{F}_{\mathbf{e x t}}$ and $\mathbf{F}_{\text {int }}$ contain the influence of the passing train and the internal forces, respectively.

As it uses high-order polynomials, the method is exponentially accurate: an increase of the polynomial order leads to an exponential decrease of the error. This property, called spectral precision, gives its name to the method. Using under-integration, the mass matrix becomes naturally diagonal, which allows to use an explicit second-order finitedifference scheme in time. Even though the stability condition requires to use very small time steps, the construction of the solution at each time step is very easy because the inversion of the mass matrix is instantaneous. This feature does not occur in classical high-order FEM. Our implementation of the SEM has demonstrated scalability for more than 10 '000 cores [39] while other implementations in the literature have even been shown to scale over 100 '000 cores [50].

\subsection{Influence of heterogeneity of the ballast on the wavefield}

To discuss the influence of heterogeneity of the ballast on the wave fields induced by the passage of a train, we consider two different models, and compare them. The models are similar in all features except for the mechanical parameters in the ballast layer: one of the model has homogeneous properties in the ballast, while the other is heterogeneous. Note that the average value of the heterogeneous Young's modulus is equal to the value of the homogeneous Young's modulus. Note also that the input force is normalized a posteriori in order to make sure that the total energy introduced in the two models is the same.

The displacement field at time $t=0.27 \mathrm{~s}$ in the case when the ballast is homogeneous is plotted at the top of Fig. 22. The pattern is quite simple, with an energy clearly concentrated under the moving load. There seems to be a guided wave within the ballast layer, connecting with Rayleigh wave in the soil. At the bottom of Fig. 22, the same displacement field is plotted in the case of the heterogeneous ballast. The wave pattern is very different, with most of the energy remaining within the ballast, and not necessarily concentrated below the moving load. The energy seems to be trapped within the ballast layer, and not propagating forward as in the case of guided waves. This localization of the energy, known as Anderson localization [6], is due to the strong heterogeneity of the material properties, and eventually leads to a lower radiation of energy towards the sensors. For the sensors in the free field, amplitudes are therefore small, which may be mistaken for damping or dissipation.

Note that there is no energy dissipation in our model, so that the later part of the recordings is probably not very realistic. Within an actual ballast, a large part of the energy trapped in the ballast layer would be dissipated by friction and crushing of grains. To incorporate this dissipation in a generalized model requires developing a continuum dissipation model adequate for granular materials, with parameters homogeneous in space or not [46]. Even though there is no dissipation, sensors located in the free field would still feel 


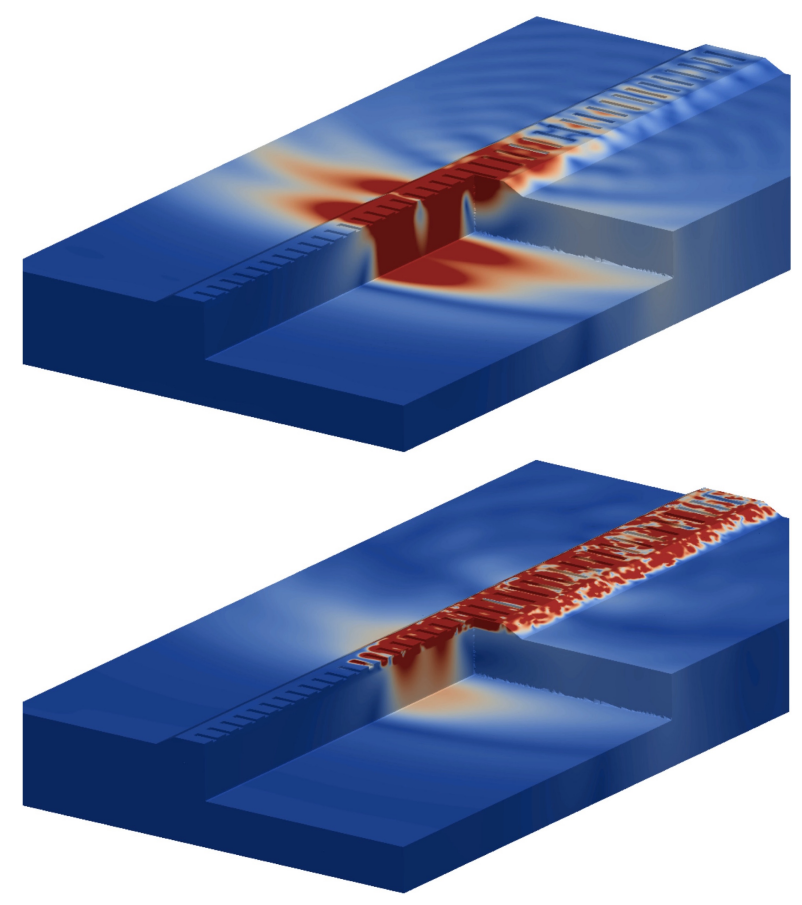

Fig. 22 Displacement fields at time $t=0.27 \mathrm{~s}$. The upper figure corresponds to the simulation with homogeneous ballast, while the lower one corresponds to simulation with heterogeneous ballast.

apparent damping because most of the energy does not escape the ballast, resulting in smaller displacements of those sensors.

The fact that the two images in Fig. 22 are so different is a clear demonstration that heterogeneity in the ballast should be taken into account. Indeed, the results obtained here can be shown to be much more compatible with actual observations made in the field [26] than previous models.

\section{Conclusions}

In this paper, we introduced a novel approach to the modeling of ballasted railway tracks. In between homogeneous continuum models, that cannot reproduce the complex dynamical behavior of realistic tracks, and discontinuous models that are difficult to simulate at the appropriate scale, we proposed a heterogeneous continuum model. Young's modulus was modeled as a randomly-fluctuating parameter whose statistics have been identified on discrete simulations of granular samples with realistic shapes. The identified stress distributions match extremely well the equivalent stresses in the discrete simulations, even for components that have not been used in the identification process. The impact is enormous on the wave field produced by the passage of a train on a typical ballasted railway track, so that this heterogeneity should clearly be investigated further.

Although the first results are extremely encouraging, several aspects should be improved. Only Young's modulus has been modeled as random here, whereas it might be more appropriate to model the entire constitutive tensor as random, potentially introducing anisotropy [100], and certainly considering constitutive non-linearities [46]. It would be interesting to discuss in detail the impact of the heterogeneity on wave propagation velocities, as this feature is classically observed in wave propagation in granular media.

In terms of limitations of the model, it should be stressed that the model can obviously not take into account situations when grains re-arrange. It is therefore not appropriate for fatigue simulations or long-term behavior of the track. Also, it considers locally averaged stresses and strains, so that it is probably not appropriate to predict local stress states, sometimes required for fatigue simulation at the interface with the sleepers for instance.

Finally, it should be reminded that the identification process was performed in statics. More realistic or predictive simulations would require the inverse problem to be performed on dynamical measurements. This is currently under process, using experimental campaigns on French railway tracks, and can hopefully be introduced soon.

\section{References}

1. Logiciel de mécanique gérant les contacts. URL https://gitxen.lmgc.univ-montp2.fr/lmgc90/lmgc90_user/wikis/home

2. Agnolin, I., Roux, J.N.: On the elastic moduli of threedimensional assemblies of spheres: characterization and modeling of fluctuations in the particle displacement and rotation. Int. J. Solids Struct. 45(3-4), 1101-1123 (2008). DOI 10.1016/j.ijsolstr.2007.07.016

3. Ahmed, S., Harkness, J., Le Pen, L., Powrie, W., Zervos, A.: Numerical modelling of railway ballast at the particle scale. Int. J. Numer. Anal. Meth. Geomech. (2015). DOI 10.1002/nag.2424. Accepted for publication

4. Al Shaer, A., Duhamel, D., Sab, K., Foret, G., Schmitt, L.: Experimental settlement and dynamic behavior of a portion of ballasted railway track under high speed trains. Journal of Sound and Vibration 316(March), 211-233 (2008). DOI 10.1016/j.jsv.2008.02.055

5. Alart, P., Iceta, D., Dureisseix, D.: A nonlinear domain decomposition formulation with application to granular dynamics. Comp. Methods Appl. Mech. Engrg. 205-208, 59-67 (2012). DOI 10.1016/j.cma.2011.04.024

6. Anderson, P.W.: Absence of Diffusion in Certain Random Lattices. Physical Review 109(5), 1492-1505 (1958)

7. Andrianov, I.V., Awrejcewicz, J., Weichert, D.: Improved continuous models for discrete media. Math. Prob. Engrg. 2010 986,242 (2010). DOI 10.1155/2010/986242

8. Arnst, M., Ghanem, R.G.: Probabilistic equivalence and stochastic model reduction in multiscale analysis. Comp. Methods Appl. Mech. Engrg. 197, 3584-3592 (2008). DOI 10.1016/j.cma.2008.03.016

9. Azéma, E., Radjaï, F.: Stress-strain behavior and geometrical properties of packings of elongated particles. Physical Review E 81(5), 051,304 (2010)

10. Azéma, E., Radjai, F., Dubois, F.: Packings of irregular polyhedral particles: Strength, structure, and effects of angularity. Physical Review E 87(6), 062,203 (2013) 
11. Azéma, E., Radjai, F., Saussine, G.: Quasistatic rheology, force transmission and fabric properties of a packing of irregular polyhedral particles. Mech. Mater. 41, 729-741 (2009). DOI 10.1016/j.mechmat.2009.01.021

12. Bagi, K.: Statistical analysis of contact force components in random granular assemblies. Granular Matter 5(1), 45-54 (2003). DOI 10.1007/s10035-002-0123-5

13. Bagi, K.: Analysis of microstructural strain tensors for granular assemblies. Int. J. Solids Struct. 43(10), 31,666-3184 (2006). DOI 10.1016/j.ijsolstr.2005.07.016

14. Bardet, J.P., Vardoulakis, I.: The asymetry of stress in granular media. Int. J. Solids Struct. 38(2), 353-367 (2001). DOI 10.1016/S0020-7683(00)00021-4

15. Blum, L., Stell, G.: Polydisperse systems. I. scattering function for polydisperse fluids of hard or permeable spheres. J. Chem. Phys. 71(1), 42-46 (1979). DOI 10.1063/1.438088

16. Blum, L., Stell, G.: Erratum: Polydisperse systems. I. scattering function for polydisperse fluids of hard or permeable spheres. J. Chem. Phys. 72(3), 2212 (1980). DOI 10.1063/1.439328

17. Brandt, H.: A study of the speed of sound in porous granular media. J. Appl. Mech. 22, 479-186 (1955)

18. Cambou, B., Chaze, M., Dedecker, F.: Change of scale in granular materials. Europ. J. Mech. A/Solids 19(6), 999-1014 (2000). DOI 10.1016/S0997-7538(00)01114-1

19. Chang, C.S., Chao, S.J., Chang, Y.: Estimates of elastic moduli for granular material with anisotropic random packing structure. Int. J. Solids Struct. 32(14), 1989-2008 (1995). DOI 10.1016/0020-7683(94)00225-L

20. Chang, C.S., Lun, M.: Elastic material constants for isotropic granular solids with particle rotation. Int. J. Solids Struct. 29(8), 1001-1018 (1992). DOI 10.1016/0020-7683(92)90071-Z

21. Cohen, G.: Higher-order numerical methods for transient wave equations. Scientific Computation. Springer (2001)

22. Connolly, D.P., Kouroussis, G., Laghrouche, O., Ho, C.L., Forde, M.C.: Benchmarking railway vibrations - track, vehicle, ground and building effects. Constr. Build. Mater. (2014). DOI 10.1016/j.conbuildmat.2014.07.042. In press

23. Coopersmith, S.N., Liu, C.H., Majumdar, S., Narayan, O., Witten, T.A.: Model for force fluctuations in bead packs. Phys. Rev. E 53(5), 4673-4685 (1996). DOI 10.1103/PhysRevE.53.4673

24. Cundall, P.A., Strack, O.D.: A discrete numerical model for granular assemblies. Geotechnique 29(1), 47-65 (1979). DOI 10.1680/geot.1979.29.1.47

25. d'Aguanno, B., Klein, R.: Integral-equation theory of polydisperse Yukawa systems. Phys. Rev. A 46(12), 7652-7656 (1992). DOI 10.1103/PhysRevA.46.7652

26. de Abreu Corrêa, L., Cottereau, R., Bongini, E., Costa d'Aguiar, S., Faure, B., Voivret, C.: Impact of the heterogeneity of the ballast on the dynamical behavior of the ballast-soil system. In: Proceedings of the CM3 Conference on Computational Transport. Springer (2016). Submitted for publication

27. Digby, P.J.: The effective elastic moduli of porous granular rocks. J. Appl. Mech. 48(4), 803-808 (1981). DOI 10.1115/1.3157738

28. Drescher, A., de Josselin de Jong, G.: Photoelastic verification of a mechanical model for the flow of a granular material. J. Mech. Phys. Solids 20(5), 337-340 (1972). DOI 10.1016/00225096(72)90029-4

29. Durán, O., Kruyt, N.P., Luding, S.: Analysis of three-dimensional micro-mechanical strain formulations for granular materials: evaluation of accuracy. Int. J. Solids Struct. 47(2), 251-260 (2010). DOI 10.1016/j.ijsolstr.2009.09.035

30. Eloy, C., Clément, E.: Stochastic aspects of the force network in a regular granular piling. J. Phys. I 7(12), 1541-1558 (1997). DOI 10.1051/jp1:1997155

31. Evesque, P.: Distribution of contact forces in a homogeneous granular material of identical spheres under triaxial compression. Poudres \& Grains 14(4), 82-95 (2004)
32. Festa, G., Vilotte, J.P.: The Newmark scheme as velocitystress time-staggering: An efficient PML implementation for spectral element simulations of elastodynamics. Geophysical Journal International 161(3), 789812 (2005). DOI 10.1111/j.1365-246X.2005.02601.x. URL http://gji.oxfordjournals.org/cgi/doi/10.1111/j.1365246X.2005.02601.X

33. GDR MiDi: On dense granular flows. Europ. Phys. J. E: Soft Matter 14(4), 341-65 (2004). DOI 10.1140/epje/i2003-10153-0

34. Goddard, J.D.: Nonlinear elasticity and pressure-dependent wave speeds in granular media. Proc. Royal Soc. A 430(1878), 105131 (1990). DOI 10.1098/rspa.1990.0083

35. Goldhirsch, I., Goldenberg, C.: On the microscopic foundations of elasticity. European Physical Journal E 9(3), 245-251 (2002). DOI 10.1140/epje/i2002-10073-5

36. Griffith, W.L., Triolo, R., Tampere, A.L.: Analytical structure function of a polydisperse Percus-Yevick fluid with Schulz (gamma) distributed diameters. Phys. Rev. A 33(3), 2197-2200 (1986). DOI 10.1103/PhysRevA.33.2197

37. Grigoriu, M.: Simulation of stationary non-gaussian translation processes. J. Engng. Mech. 124(2), 121-126 (1998)

38. Guerin, N.: Approche expérimentale et numérique du comportement du ballast des voies ferrées. Ph.D. thesis, École nationale des ponts et chaussées (1996). In French

39. Guillot, L., Aubry, L., Le Piver, F., Mariotti, C., Sèbe, O., Thauvin, E., Odonbaatar, C., Ulziibat, M., Demberel, S., Sukhbaatar, S.: Numerical simulation of seismic wave propagation: site effects. Chocs 45, 29-36 (2014)

40. Han, F., Cui, J., Yu, Y.: The statistical second-order two-scale method for mechanical properties of statistically inhomogeneous materials. Int. J. Numer. Meth. Engrg. 84, 972-988 (2010). DOI 10.1002/nme. 2928

41. Heckl, M., Hauck, G., Wettschureck, R.: Structure-borne sound and vibration from rail traffic. J. Sound Vib. 193(1), 175-184 (1996). DOI 10.1006/jsvi.1996.0257

42. Howell, D.W., Behringer, R.P., Veje, C.T.: Fluctuations in granular media. Chaos 9(3), 559-572 (1999). DOI 10.1063/1.166430

43. Hutter, K., Rajagopal, K.R.: On flows of granular materials. Cont. Mech. Thermodyn. 6, 81-139 (1994)

44. Indraratna, B., Salim, W., Rujikiatkamjorn, C.: Advanced rail geotechnology. Ballasted track. CRC Press (2011)

45. INNOTRACK D2.1.3: First phase on the modelling of poor quality sites. Tech. Rep. Project no. tip5 ct-2006-031415, European commission - sixth framework program (2009)

46. Jehel, P., Cottereau, R.: On damping created by heterogeneous yielding in the numerical analysis of nonlinear RC frame elements. Comp. \& Struct. 154, 192-203 (2015). DOI 10.1016/j.compstruc.2015.03.001

47. Jenkins, J., Johnson, D., La Ragione, L., Makse, H.: Fluctuations and the effective moduli of an isotropic, random aggregate of identical, frictionless spheres. J. Mech. Phys. Solids 53(1), 197225 (2005). DOI 10.1016/j.jmps.2004.06.002

48. Jop, P., Forterre, Y., Pouliquen, O.: A constitutive law for dense granular flows. Nature 441(7094), 727-30 (2006). DOI 10.1038/nature04801

49. Kincaid, J.M., Weis, J.J.: Radial distribution function of a hardsphere solid. Molecular Phys. 34(4), 931-938 (1977). DOI 10.1080/00268977700102241

50. Komatitsch, D.: The spectral-element method in seismology. Geophysical Monograph Series 157(55), 205-227 (2005). DOI 10.1029/157GM13

51. Kuhn, M.R.: Structured deformation in granular materials. Mech. Mater. 31(6), 407-429 (1999). DOI 10.1016/S01676636(99)00010-1

52. Lado, F.: Integral equation theory of polydisperse colloidal suspensions using orthogonal polynomial expansions. Phys. Rev. E 54(4), 4411-4419 (1996). DOI 10.1103/PhysRevE.54.4411 
53. Lagarias, J.C., Reeds, J.A., Wright, M.H., Wright, P.E.: Convergence properties of the nelder-mead simplex method in low dimensions. SIAM Journal on optimization 9(1), 112-147 (1998)

54. Leibig, M.: Model for the propagation of sound in granular materials. Phys. Rev. E 49(2), 1647-1656 (1994). DOI 10.1103/PhysRevE.49.1647

55. Lim, W.L., McDowell, G.R.: Discrete element modelling of railway ballast. Granular Matter 7(1), 19-29 (2005). DOI 10.1007/s10035-004-0189-3

56. Liu, C.H., Nagel, S.R., Schecter, D.A., Coopersmith, S.N., Majumdar, S., Narayan, O., Witten, T.A.: Force fluctuations in bead packs. Sci. 269(5223), 513-515 (1995). DOI $10.1126 /$ science. 269.5223 .513

57. Lombaert, G., Degrande, G., Kogut, J., François, S.: The experimental validation of a numerical model for the prediction of railway induced vibrations. J. Sound Vib. 297(3-5), 512-535 (2006). DOI 10.1016/j.jsv.2006.03.048

58. Love, A.: A Treatise on the Mathematical Theory of Elasticity. No. v. 4 in A Treatise on the Mathematical Theory of Elasticity. Cambridge University Press (1927)

59. Lu, M., McDowell, G.R.: The importance of modelling ballast particle shape in the discrete element method. Granular Matter 9, 69-80 (2007). DOI 10.1007/s10035-006-0021-3

60. Makse, H.A., Gland, N., Johnson, D.L., Schwartz, L.: Granular packings: nonlinear elasticity, sound propagation, and collective relaxation dynamics. Phys. Rev. E 70(6), 061,302 (2004). DOI 10.1103/PhysRevE.70.061302

61. McNamara, S., Flekkøy, E.G., Måløy, K.J.: Grains and gas flow: molecular dynamics with hydrodynamic interactions. Phys. Rev. E 61(4), 4054-4059 (2000). DOI 10.1103/PhysRevE.61.4054

62. Miller, B., O'Hern, C., Behringer, R.P.: Stress fluctuations for continuously sheared granular materials. Phys. Rev. Lett. 77(15), 3110-3113 (1996). DOI 10.1103/PhysRevLett.77.3110

63. Modaressi, A., Boufellouh, S., Evesque, P.: Modeling of stress distribution in granular piles: Comparison with centrifuge experiments. Chaos 9(3), 523-543 (1999). DOI 10.1063/1.166427

64. Moreau, G., Caillerie, D.: Continuum modeling of lattice structures in large displacement applications to buckling analysis. Comp. \& Struct. 68, 181-189 (1998). DOI 10.1016/S00457949(98)00041-8

65. Moreau, J.J.: Unilateral contact and dry friction in finite freedom dynamics. In: J. Moreau, P.D. Panagiotopoulos (eds.) Nonsmooth mechanics and applications, CISM Courses and Lectures, vol. 302, pp. 1-81 (1989)

66. Moreau, J.J.: Numerical investigation of shear zones in granular materials. In: P. Grassberger, D. Wolf (eds.) Proceedings of HLRZ-workshop on friction, arching, contact dynamics, pp. 233-247. World Scientific (1997)

67. Moreau, J.J.: The stress tensor in granular media and in other mechanical collections. In: B. Cambou, M. Jean, F. Radjaï (eds.) Micromechanics of granular materials. Wiley (2001)

68. Mueth, D.M., Jaeger, H.M., Nagel, S.R.: Force distribution in a granular medium. Phys. Rev. E 57(3), 3164-3169 (1998). DOI 10.1103/PhysRevE.57.3164

69. Nguyen, N.S., Magoariec, H., Cambou, B.: Local stress analysis in granular materials at a mesoscale. Int. J. Numer. Anal. Meth. Geomech. 36, 1609-1635 (2012). DOI 10.1002/nag.1063

70. Nichol, K., Zanin, A., Bastien, R., Wandersman, E., van Hecke, M.: Flow-induced agitations create a granular fluid. Phys. Rev. Lett. 104(7) (2009). DOI 10.1103/PhysRevLett.104.078302

71. Nicodemi, M.: Force correlations and arch formation in granular assemblies. Phys. Rev. Lett. 80(6), 1340-1343 (1998). DOI 10.1103/PhysRevLett.80.1340

72. Nicot, F., Hadda, N., Darve, F.: Second-order work analysis for granular materials using a multiscale approach. Int. J. Numer. Anal. Meth. Geomech. 37(17), 2987-3007 (2013). DOI 10.1002/nag.2175
73. Norris, A.N., Johnson, D.L.: Nonlinear elasticity of granular media. J. Appl. Mech. 64(1), 39-49 (1997). DOI $10.1115 / 1.2787292$

74. Ostoja-Starzewski, M.: Lattice models in micromechanics. Appl. Mech. Rev. 55(1), 35-60 (2002). DOI 10.1115/1.1432990

75. Paludo, L., Bouvier, V., de Abreu Correa, L., Cottereau, R., Clouteau, D.: Efficient parallel generation of random field of mechanical properties for geophysical application. In: 6th International Conference on Earthquake Geotechnical Engineering. Christchurch, New Zealand (2015)

76. Paolucci, R., Maffeis, A., Scandella, L., Stupazzini, M., Vanini, M.: Numerical prediction of low-frequency ground vibrations induced by high-speed trains at Ledsgaard, Sweden. Soil Dynamics Earthquake Engr. 23(6), 425-433 (2003). DOI 10.1016/S02677261(03)00061-7

77. Pasternak, E., Mühlhaus, H.B.: Generalised homogenisation procedures for granular materials. Mathematics and Mechanics of Granular Materials 52(1), 199-229 (2005). DOI 10.1007/14020-4183-7_12

78. Puig, B., Akian, J.L.: Non-gaussian simulation using Hermite polynomials expansion and maximum entropy principle. Prob. Engrg. Mech. 19(4), 293-305 (2004). DOI 10.1016/j.probengmech.2003.09.002

79. Quintanilla, J.: Microstucture functions for random media with impenetrable particles. Phys. Rev. E 60, 5788-5794 (1999). DOI 10.1103/PhysRevE.60.5788

80. Radjai, F., Delenne, J.Y., Azéma, E., Roux, S.: Fabric evolution and accessible geometrical states in granular materials. Granular Matter 14(2), 259-264 (2012)

81. Radjai, F., Jean, M., Moreau, J.J., Roux, S.: Force distributions in dense two-dimensional granular systems. Phys. Rev. Lett. 77(2), 274-277 (1996). DOI 10.1103/PhysRevLett.77.274

82. Radjai, F., Roux, S., Moreau, J.: Contact forces in a granular packing. Chaos 9(3), 544-550 (1999). DOI 10.1063/1.166428

83. Radjai, F., Topin, V., Richefeu, V., Voivret, C., Delenne, J.Y., Azéma, E., El Youssoufi, M.S.: Force transmission in cohesive granular media. Mathematical Modeling and Physical Instances of granular Flows pp. 240-260 (2010)

84. Radjai, F., Wolf, D., Jean, M., Moreau, J.: Bimodal character of stress transmission in granular packings. Phys. Rev. Lett. 80, 61-64 (1998). DOI 10.1103/PhysRevLett.80.61

85. Reddy, K., Forterre, Y., Pouliquen, O.: Evidence of mechanically activated processes in slow granular flows. Phys. Rev. Lett. 106(10), 108,301 (2011). DOI 10.1103/PhysRevLett.106.108301

86. Renouf, M., Dubois, F., Alart, P.: A parallel version of the non smooth contact dynamics algorithm applied to the simulation of granular media. J. Comput. Appl. Math. 168(1-2), 375-382 (2004). DOI 10.1016/j.cam.2003.05.019

87. Rosenblatt, M.: Remarks on a multivariate transformation. The annals of mathematical statistics pp. 470-472 (1952)

88. Rothenburg, L., Bathurst, R.J.: Analytical study of induced anisotropy in idealized granular materials. Geotechnique 39, 601-614 (1989)

89. Rothenburg, L., Kruyt, N.P.: Micromechanical definition of an entropy for quasi-static deformation of granular materials. J. Mech. Phys. Solids 57(3), 634-655 (2009). DOI 10.1016/j.jmps.2008.09.018

90. Saint-Cyr, B., Delenne, J.Y., Voivret, C., Radjai, F., Sornay, P.: Rheology of granular materials composed of nonconvex particles. Physical Review E 84(4), 041,302 (2011)

91. Satake, M.: Some considerations on the mechanics of granular materials. In: E. Kröner (ed.) Proceedings of the IUTAM Symposium on the Generalized Cosserat Continuum and the Continuum Theory of Dislocations with Applications, pp. 156-159 (1968). DOI 10.1007/978-3-662-30257-6_19 
92. de Saxcé, G., Fortin, J., Millet, O.: About the numerical simulation of the dynamics of granular media and the definition of the mean stress tensor. Mechanics of Materials 36, 1175-1184 (2004). DOI 10.1016/j.mechmat.2003.01.002

93. Serero, D., Reydellet, G., Claudin, P., Clément, E., Levine, D.: Stress response function of a granular layer: quantitative comparison between experiments and isotropic elasticity. Europ. J. Phys. E 6(2), 169-179 (2001). DOI 10.1007/s101890170019

94. Shin, H., Santamarina, J.: Role of particle angularity on the mechanical behavior of granular mixtures. J. Geotech. Geoenv. Engrg. 139(2), 353-355 (2013). DOI 10.1061/(ASCE)GT.19435606.0000768

95. Shinozuka, M., Deodatis, G.: Simulation of stochastic processes by spectral representation. Appl. Mech. Rev. 44(4), 191-204 (1991). DOI 10.1115/1.3119501

96. Shinozuka, M., Deodatis, G.: Simulation of multi-dimensional gaussian stochastic fields by spectral representation. Applied Mechanics Reviews 49, 29-53 (1996)

97. Soize, C.: Non-Gaussian positive-definite matrix-valued random fields for elliptic stochastic partial differential operators. Comp. Methods Appl. Mech. Engrg. 195(1-3), 26-64 (2006). DOI 10.1016/j.cma.2004.12.014

98. Staron, L., Radjai, F., Vilotte, J.P.: Granular micro-structure and avalanche precursors. J. Stat. Mech.: Theory Exp. 07(P07014) (2006). DOI 10.1088/1742-5468/2006/07/P07014

99. Suiker, a.S.J., De Borst, R., Chang, C.S.: Micro-mechanical modelling of granular material. Part 1: Derivation of a secondgradient micro-polar constitutive theory. Acta Mechanica 149, 161-180 (2001). DOI 10.1007/BF01261670

100. Ta, Q.A., Clouteau, D., Cottereau, R.: Modeling of random anisotropic elastic media and impact on wave propagation. Europ. J. Comput. Mech. 19(1-3), 241-253 (2010). DOI 10.3166/ejcm.19.241-253

101. Thi Minh Phuong, H., Alart, P., Dureisseix, D., Saussine, G.: A domain decomposition method for granular dynamics using discrete elements and application to railway ballast. Ann. Solid Struct. Mech. 2(2-4), 87-98 (2011). DOI 10.1007/s12356-0110020-x

102. Tollenaere, H., Caillerie, D.: Continuous modeling of lattice structures by homogenization. Adv. Engrg. Software 29(7-9), 699-705 (1998). DOI 10.1016/S0965-9978(98)00034-9

103. Torquato, S.: Random heterogeneous materials. Microstructure and macroscopic properties. Springer (2001)

104. Torquato, S., Stell, G.: Microstructure of two-phase random media. V. the n-point matrix probability functions for impenetrable spheres. J. Chem. Phys. 82, 980-987 (1985). DOI $10.1063 / 1.448475$

105. Tsoungui, O., Vallet, D., Charmet, J.C.: Use of contact area trace to study the force distributions insisde 2D granular systems. Granular Matter 1(2), 65-69 (1998). DOI $10.1007 / \mathrm{s} 100350050010$

106. Tsuchikura, T., Satake, M.: Statistical measure tensors and their application to computer simulation analysis of biaxial compression text. In: H. Murakami, J.E. Luco (eds.) Engineering Mechanics: a Force for 21st Century, pp. 1732-1735. ASCE (1998)

107. Verlet, L., Weis, J.J.: Equilibrium theory of simple liquids. Phys. Rev. A 5(2), 939-952 (1972). DOI 10.1103/PhysRevA.5.939

108. Voivret, C.: Texture et comportement des matériaux granulaires à grande polydispersité. Ph.D. thesis, Université Montpellier 2 (2008)

109. Vrij, A.: Mixtures of hard spheres in the Percus-Yevick approximation. light scattering at finite angles. J. Chem. Phys. 71(8), 3267-3270 (1979). DOI 10.1063/1.438756

110. Walton, K.: The effective elastic moduli of a random packing of spheres. J. Mech. Phys. Solids 35(2), 213-226 (1987). DOI 10.1016/0022-5096(87)90036-6
111. Weber, J.: Recherches concernant les contraintes intergranulaires dans les milieux pulvérulents. Bulletin de liaison des Ponts et Chaussées 20, 1-20 (1966) 